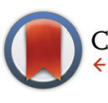

CrossMark \& click for updates

Cite this: Dalton Trans., 2016, 45 18229

Received 24th June 2016, Accepted 24th October 2016 DOI: $10.1039 / c 6 d t 02541 b$ www.rsc.org/dalton

\title{
Synthesis and characterization of metal-rich phosphonium polyelectrolytes and their use as precursors to nanomaterials $\uparrow$
}

\begin{abstract}
Amir Rabiee Kenaree and Joe B. Gilroy*
Upon efficient quaternization and salt metathesis of stable triethyl ferrocene/ruthenocene phosphines, styrene-based phosphonium triflate monomers with four different stoichiometric ratios of Fe/Ru were synthesized. Free-radical polymerization of the monomers afforded four polyelectrolytes $\left(M_{n}: 38650-69100 \mathrm{~g} \mathrm{~mol}^{-1}, \oplus: 3.16-4.10\right)$ that retained many of the spectroscopic and electrochemical properties of the ferrocene/ruthenocene units. TGA studies demonstrated the thermal stability (onset of decomposition: $310{ }^{\circ} \mathrm{C}$ ) and high char yields $\left(33-54 \%\right.$ at $1000{ }^{\circ} \mathrm{C}$ ) of the polyelectrolytes. Pyrolysis in $\mathrm{N}_{2} / \mathrm{H}_{2}$ (95/5) (film thickness of $\sim 6 \mu \mathrm{m}, 1000{ }^{\circ} \mathrm{C}, 3 \mathrm{~h}$ ) yielded crystalline, mixed-phase nanomaterials containing iron, ruthenium, and phosphorus with compositions influenced by the structure of the parent polyelectrolytes.
\end{abstract}

\section{Introduction}

Metallopolymers, which differ from coordination polymers that have dynamic structures, are an intriguing class of materials that benefit from the processability of macromolecules and functional properties of transition metals. ${ }^{1}$ To date, many metallopolymers have been successfully synthesized and utilized as redox-active, catalytic, emissive, biomedical, and magnetic materials. ${ }^{2}$ However, examples of heterobimetallic polymers, which can take advantage of the properties of more than one type of metal, are far less common.

There are several existing strategies for the incorporation of more than one type of transition metal into polymer structures. For example, post-polymerization functionalization of metallopolymers can be employed for the addition of transition metals to the repeating unit of the polymer backbone. ${ }^{3}$ However, it can be a challenge to completely functionalize all of the repeating units in the polymer backbone. Manners and co-workers have used their well-established ring-opening polymerization methodology for the

Department of Chemistry and the Centre for Advanced Materials and Biomaterials Research (CAMBR), The University of Western Ontario, 1151 Richmond St. N., London, Ontario, Canada, N6A 5B7. E-mail: joe.gilroy@uwo.ca;

Tel: +1-519-661-2111 ext. 81561

$\dagger$ Electronic supplementary information (ESI) available. CCDC 1476067. For ESI and crystallographic data in CIF or other electronic format see DOI: 10.1039/ c6dt02541b synthesis of polyferrocenylsilanes (PFSs) to prepare monometallic acetylide-substituted PFSs, which were further reacted with $\mathrm{Co}_{2}(\mathrm{CO})_{8},\left[\mathrm{MoCp}(\mathrm{CO})_{2}\right]_{2}$, and $\left[\mathrm{NiCp}(\mathrm{CO})_{2}\right]_{2}$ to produce heterobimetallic polymers $1 .^{3 a-c}$ They also showed that reactive ion etching (RIE), ${ }^{3 a}$ electron-beam lithography, ${ }^{3 b}$ and pyrolysis ${ }^{3 c}$ can be used to convert the heterobimetallic polymers produced to the corresponding bimetallic alloy nanoparticles (NPs).

Copolymerization of more than one type of metal-containing monomer is another strategy that can yield heterobimetallic polymers, where the metal ratio can be adjusted by controlling the ratio of the repeating units. ${ }^{4}$ Following this strategy and starting with two methacrylate-based ferrocene- and cobaltocenium-containing monomers, the Tang group recently performed a reversible addition-fragmentation chain-transfer (RAFT) polymerization and successfully synthesized heterobimetallic diblock copolymers containing cobaltocenium and ferrocene units 2 . By pyrolysis of the heterobimetallic copolymer under $\mathrm{N}_{2} / \mathrm{H}_{2}$, magnetic nanomaterials comprised of $\mathrm{Fe}_{x} \mathrm{Co}_{y} \mathrm{P}$ (where $x+y=2$ ) were realized. ${ }^{4 e}$

Starting with a monomer that has more than one metal within its structure is a strategy that affords heterobimetallic polymers with transition metal content equal to that of the monomer. ${ }^{5}$ For instance, Wong and co-workers synthesized an iron- and platinum-containing heterobimetallic polymer and used nanoimprint lithography to generate nanopatterns of the resulting polymer 3. RIE converted the polymer to nanopatterned magnetic Fe/Pt NPs. ${ }^{5 c}$ The Manners group has also reported the synthesis of a palladium-based [1]ferrocenophane 4 that was thermolized at $190{ }^{\circ} \mathrm{C}$ under vacuum to directly 


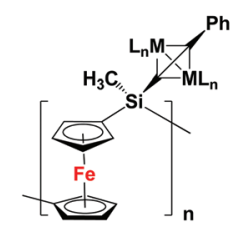

$\mathrm{ML}_{\mathrm{n}}=\mathrm{NiCp}, \mathrm{Co}(\mathrm{CO})_{3}, \mathrm{MoCp}(\mathrm{CO})_{2}$

1
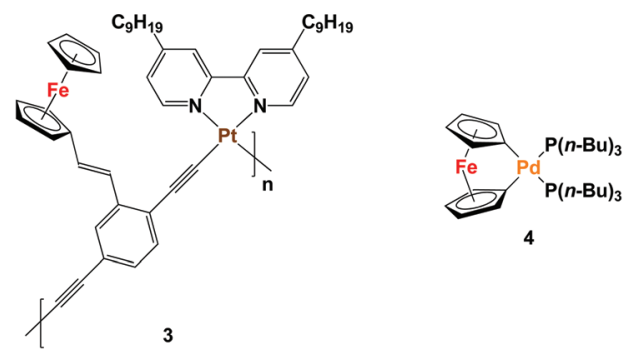

yield Fe/Pd alloy NPs, presumably via a heterobimetallic polymer. $^{5} e$

Bimetallic particles are an interesting subclass of nanomaterials that benefit from their high surface area. ${ }^{6}$ For example, $\mathrm{Fe} / \mathrm{Ru}$ heterobimetallic particles are industrially valuable materials due to their catalytic role in processes including hydrogenation, ${ }^{7}$ the water-gas shift reaction, ${ }^{8}$ and the FischerTropsch synthesis. ${ }^{9}$ They are conventionally prepared by techniques such as thermolysis and co-reduction of metal ions. ${ }^{10}$ Although, metallopolymers can serve as precursors to metalcontaining nanomaterials, ${ }^{11}$ few reports of the generation of bimetallic nanomaterials from heterobimetallic polymers have been made. To the best of our knowledge Fe/Ru nanomaterials have not been prepared via the degradation of heterobimetallic polymers.

Herein, we describe our efforts to address this deficiency. Specifically, we have prepared polyelectrolytes based on phosphonium scaffolds containing ethylferrocene and ethylruthenocene units (Fe/Ru: 3/0, 2/1, 1/2, 0/3) and explored their preceramic properties by conducting pyrolysis experiments under a reducing atmosphere.

\section{Experimental section}

\section{General considerations}

Reactions and manipulations were carried out under a nitrogen atmosphere using standard glove box or Schlenk techniques unless otherwise stated. Solvents were obtained from Caledon Laboratories and Fischer Scientific, dried using an Innovative Technologies Inc. solvent purification system, collected under vacuum, and stored under a nitrogen atmosphere over $4 \AA$ molecular sieves. Reagents were purchased from Sigma-Aldrich or Alfa Aesar and used as received, aside from 4-vinylbenzyl chloride which was purified according to a literature procedure and stored under $\mathrm{N}_{2}$ at $-35{ }^{\circ} \mathrm{C} .{ }^{12}$ Tertiary phosphines 5a-d were synthesized according to reported protocols. ${ }^{13}{ }^{1} \mathrm{H},{ }^{13} \mathrm{C}\left\{{ }^{1} \mathrm{H}\right\},{ }^{19} \mathrm{~F}$ and ${ }^{31} \mathrm{P}$ NMR spectra were recorded on a $600 \mathrm{MHz}\left({ }^{1} \mathrm{H}: 599.5 \mathrm{MHz},{ }^{13} \mathrm{C}: 150.8 \mathrm{MHz},{ }^{19} \mathrm{~F}: 563.9 \mathrm{MHz}\right.$ and ${ }^{31} \mathrm{P}$ : $242.6 \mathrm{MHz}$ ) Varian INOVA instrument. ${ }^{1} \mathrm{H}$ NMR spectra were referenced to residual $\left(\mathrm{CD}_{3}\right)\left(\mathrm{CD}_{2} \mathrm{H}\right) \mathrm{SO}(2.50 \mathrm{ppm})$ and ${ }^{13} \mathrm{C}\left\{{ }^{1} \mathrm{H}\right\}$ NMR spectra were referenced to DMSO- $d_{6}$ (39.5 ppm). ${ }^{31} \mathrm{P}$ NMR spectra were referenced to $\mathrm{PPh}_{3}$ as an internal standard $\left(-6.0 \mathrm{ppm}\right.$ relative to $\left.\mathrm{H}_{3} \mathrm{PO}_{4}\right)$. Mass spectrometry data were recorded in positive-ion mode using a Micromass/Waters Q-TOF Ultima LC-MS/MS system. UV-vis absorption spectra were recorded using a Cary 300 Scan instrument. Infrared spectra were recorded using a PerkinElmer Spectrum Two FT-IR spectrometer as thin films on $\mathrm{KBr}$ plates. Elemental analyses ( $\mathrm{C}$ and $\mathrm{H}$ ) were carried out by Laboratoire d'Analyse Élémentaire de l'Université de Montréal, Montréal, QC, Canada.

\section{Cyclic voltammetry}

Cyclic voltammograms were collected using a Bioanalytical Systems Inc. (BASi) Epsilon potentiostat and analyzed using BASi Epsilon software. Typical electrochemical cells consisted of a three-electrode setup including a glassy carbon working electrode, platinum wire counter electrode, and silver wire pseudo-reference electrode. $1 \mathrm{mM}$ degassed solutions of monomers 7a-d, combined with supporting electrolyte (0.1 M $[n$ $\left.\left.\mathrm{Bu}_{4} \mathrm{~N}\right][\mathrm{OTf}]\right)$, in a $\mathrm{CH}_{2} \mathrm{Cl}_{2} / \mathrm{CH}_{3} \mathrm{CN}(2 / 1)$ solvent mixture were prepared and run at a scan rate of $250 \mathrm{mV} \mathrm{s}^{-1}$ under a blanket of argon. To study the electrochemical behavior of the polymers, different solvents such as THF, DMF, $\mathrm{CH}_{3} \mathrm{CN}$, and $\mathrm{CH}_{2} \mathrm{Cl}_{2}$ containing $0.1 \mathrm{M}\left[n-\mathrm{Bu}_{4} \mathrm{~N}\right][\mathrm{OTf}]$ were used to make $0.2 \mathrm{mM}$ solutions of the analytes. In each case, severe plating of the oxidized forms of $\mathbf{8 a - d}$ was observed on the glassy carbon working electrode. Therefore, a 2/1 solvent mixture of $\mathrm{CH}_{2} \mathrm{Cl}_{2}$ and $\mathrm{CH}_{3} \mathrm{CN}$ was used as it was the least problematic combination. Degassed solutions of polyelectrolytes $\mathbf{8 a}-\mathbf{d}$ in a $\mathrm{CH}_{2} \mathrm{Cl}_{2} / \mathrm{CH}_{3} \mathrm{CN}(2 / 1)$ solvent mixture were prepared by stirring the mixture overnight at $40{ }^{\circ} \mathrm{C}$. After addition of the supporting electrolyte, the mixtures were sonicated for $20 \mathrm{~s}$, filtered (Nylon membrane, $0.22 \mu \mathrm{m}$ ) and using these solutions electrochemical studies were performed at a scan rate of $250 \mathrm{mV} \mathrm{s}^{-1}$ under a blanket of argon. Cyclic voltammograms were referenced relative to a decamethylferrocene internal standard ( $1 \mathrm{mM},-520 \mathrm{mV}$ relative to ferrocene/ferrocenium under identical conditions) and corrected for internal cell resistance using the BASi Epsilon software.

\section{X-ray diffraction studies}

Single crystals of monomer 7a suitable for X-ray diffraction studies were grown by slow evaporation of a THF solution. The sample was mounted on a MiTeGen polyimide micromount with a small amount of Paratone $\mathrm{N}$ oil. X-ray diffraction measurements were made on a Bruker Kappa Axis Apex2 diffractometer at a temperature of $110 \mathrm{~K}$. Initial indexing indicated that the sample crystal was non-merohedrally twinned. The twin law was determined to be:which represents a $179.8^{\circ}$ rotation about [100]. The twin fraction was included in the 
refinement as an adjustable parameter (vide infra). The unit cell dimensions were determined from a symmetry constrained fit of 5386 reflections with $6.58^{\circ}<2 \theta<47.88^{\circ}$. The data collection strategy was a number of $\omega$ and $\varphi$ scans which collected data up to $53.538^{\circ}(2 \theta)$. The frame integration was performed using SAINT. ${ }^{14}$ The resulting raw data was scaled and absorption corrected using a multi-scan averaging of symmetry equivalent data using TWINABS. ${ }^{15}$

$\begin{array}{rrr}0.99635 & 0.00646 & 0.00848 \\ 0.00835 & -1.00086 & 0.00433 \\ 0.85236 & -0.00198 & -0.99547\end{array}$

The structure was solved by using a dual space methodology using the SHELXT program. ${ }^{16}$ All non-hydrogen atoms were obtained from the initial solution. The hydrogen atoms were introduced at idealized positions and were allowed to ride on the parent atom. The twin fraction refined to a value of 0.465(1). The structural model was fit to the data using full matrix least-squares based on $F^{2}$. The calculated structure factors included corrections for anomalous dispersion from the usual tabulation. The structure was refined using the SHELXL-2014 program from the SHELX suite of crystallographic software. ${ }^{17}$ Graphic plots were produced using Mercury software (version 3.3). For additional collection and refinement details, see CCDC 1476067, Table 1 and Fig. 1.

Powder X-ray diffraction (PXRD) data for nanomaterial films deposited on silicon wafers were acquired using an Inel CPS powder diffractometer with an Inel XRG 3000 generator and Inel CPS 120 detector using a $\mathrm{CuK} \alpha$ radiation source. For diffractograms, see Fig. 5 and S36-S39. $\dagger$

Table 1 Selected X-ray diffraction data collection and refinement details for $7 a$

\begin{tabular}{ll}
\hline & $7 \mathbf{a}$ \\
\hline Chemical formula & $\mathrm{C}_{46} \mathrm{H}_{48} \mathrm{~F}_{3} \mathrm{Fe}_{3} \mathrm{O}_{3} \mathrm{PS}$ \\
$\mathrm{FW}\left(\mathrm{g} \mathrm{mol}^{-1}\right)$ & 936.42 \\
Temp $(\mathrm{K})$ & 110 \\
Crystal syst. & Triclinic \\
Crystal habit & Yellow plate \\
Space group & $P \overline{1}$ \\
$\lambda(\AA)$ & 0.71073 \\
$a(\AA)$ & $12.378(6)$ \\
$b(\AA)$ & $12.619(5)$ \\
$c(\AA)$ & $14.252(7)$ \\
$\alpha\left({ }^{\circ}\right)$ & $79.298(7)$ \\
$\beta\left({ }^{\circ}\right)$ & $68.524(10)$ \\
$\gamma\left({ }^{\circ}\right)$ & $89.953(7)$ \\
$V\left(\AA^{3}\right)$ & $2030.1(17)$ \\
$Z$ & 2 \\
$\rho\left(\mathrm{g} \mathrm{cm}^{-3}\right)$ & 1.532 \\
$\mu\left(\mathrm{cm}{ }^{-1}\right)$ & 1.204 \\
$R_{1}[I>2 \sigma(I)]$ & 0.0534 \\
wR $R_{2}[I>2 \sigma(I)]$ & 0.1278 \\
$R_{1}($ all data) & 0.0917 \\
w $R_{2}($ all data $)$ & 0.1463 \\
$\mathrm{GOF}$ & 1.042
\end{tabular}

$R_{1}=\sum\left(\left|F_{\mathrm{o}}\right|-\left|F_{\mathrm{c}}\right|\right) / \sum F_{\mathrm{o}}, \mathrm{w} R_{2}=\left[\sum\left(\mathrm{w}\left(F_{\mathrm{o}}{ }^{2}-F_{\mathrm{c}}{ }^{2}\right)^{2}\right) / \sum\left(\mathrm{w} F_{\mathrm{o}}{ }^{4}\right)\right]^{1 / 2} ; \mathrm{GOF}=$ $\left[\sum\left(\mathrm{w}\left(F_{\mathrm{o}}{ }^{2}-F_{\mathrm{c}}\right)^{2}\right) /(\text { No. of reflns. }- \text { No. of params. })\right]^{1 / 2}$.

\section{Gel permeation chromatography (GPC)}

GPC experiments were performed by PolyAnalytik Inc. Canada (London, Ontario). Molecular weights and dispersities $\left(Ð=M_{\mathrm{w}} / M_{\mathrm{n}}\right)$ were obtained using a Viscotek TDA302/GPCmax gel permeation chromatograph equipped with automatic sampler, isocratic pump, injector, in-line degasser, column and detector oven $\left(60{ }^{\circ} \mathrm{C}\right)$, refractive index detector, and Viscotek Inert Series Columns: 1× Mixed Bed Low Molecular Weight (I-MBLMW, exclusion limit of $20 \mathrm{kDa}$ PS) and $1 \times$ Mixed Bed High Molecular Weight (I-MBHMW, exclusion limit of $\left.10000 \mathrm{~g} \mathrm{~mol}^{-1} \mathrm{PS}\right)$. The eluent employed was DMF $\left(60^{\circ} \mathrm{C}\right)$ con-

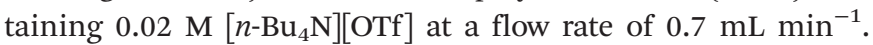
Samples were dissolved in the eluent $\left(5 \mathrm{mg} \mathrm{mL}^{-1}\right)$, heated for $1.5 \mathrm{~h}$, and filtered (Nylon membrane, $0.22 \mu \mathrm{m}$ ) before analysis. Conventional calibration of the refractive index detector was performed using a series of monodisperse polystyrene standards (PolyAnalytik). All data were processed using Viscotek's OmniSEC v4.6.2 software.

\section{Thermal analysis}

Thermal degradation studies were performed using a TA Instruments Q50 TGA instrument under an atmosphere of $\mathrm{N}_{2}$. Samples were placed in a platinum pan and heated at a rate of $10{ }^{\circ} \mathrm{C} \min ^{-1}$ from $20{ }^{\circ} \mathrm{C}$ to $1000{ }^{\circ} \mathrm{C}$ under a flow of $\mathrm{N}_{2}$

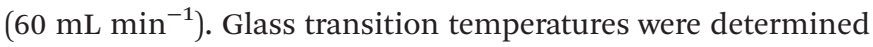
under an atmosphere of $\mathrm{N}_{2}$ using differential scanning calorimetry (DSC) on a TA Instruments DSC Q20. The polymer samples were placed in an aluminum Tzero pan and heated from room temperature to $300{ }^{\circ} \mathrm{C}$ at $10^{\circ} \mathrm{C} \mathrm{min}{ }^{-1}$ under a flow of $\mathrm{N}_{2}\left(50 \mathrm{~mL} \mathrm{~min}^{-1}\right)$ and cooled down to $0{ }^{\circ} \mathrm{C}$ at $5{ }^{\circ} \mathrm{C}$ $\min ^{-1}$, before they underwent two more heat/cool cycles. The glass transitions were determined from the second heat/cool cycle.

\section{Pyrolysis studies and scanning electron microscopy}

Films of polyelectrolytes $\mathbf{8 a - d}$ were prepared by drop-casting $250 \mu \mathrm{L}$ of a $20 \mathrm{mg} \mathrm{mL} \mathrm{m}^{-1}$ chlorobenzene solution of each polyelectrolyte onto a silicon wafer $\left(A=2.5 \mathrm{~cm}^{2}\right)$. The samples were dried in air, transferred into a vacuum oven, and allowed to dry at $50{ }^{\circ} \mathrm{C}$ for $16 \mathrm{~h}$ before they were heated at a rate of $10{ }^{\circ} \mathrm{C} \mathrm{min}^{-1}$ to a temperature of $1000{ }^{\circ} \mathrm{C}$ under a gentle flow

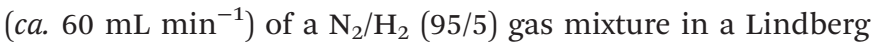
Blue $\mathrm{M}$ tube furnace. The temperature was maintained at $1000{ }^{\circ} \mathrm{C}$ for an additional $3 \mathrm{~h}$ before the furnace was cooled to room temperature at a rate of $10{ }^{\circ} \mathrm{C} \mathrm{min}^{-1}$. Polymer film thickness and the surface morphologies of thermally deposited nanomaterials on silicon wafers were assessed directly using scanning electron microscopy (SEM) at $1 \mathrm{keV}$ beam energy using LEO/Zeisss 1530 and LEO/Zeisss 1540XB instruments. Energy dispersive X-ray spectroscopy (EDX) experiments were performed at $10 \mathrm{keV}$ beam energy on the 1540XB with the equipped Oxford X-sight X-ray detector and INCA analysis software. 


\section{Representative procedure for the preparation of $6 a-d$}

Phosphonium chloride salt $6 \mathbf{6}(3 \times \mathbf{F c})$. In a sealed tube, tertiary phosphine $5 \mathbf{a}(1.00 \mathrm{~g}, 1.49 \mathrm{mmol})$ and 4-vinylbenzyl chloride ( $230 \mu \mathrm{L}, 1.63 \mathrm{mmol}, 1.1$ equiv.) were combined with $\mathrm{DMF} / \mathrm{THF}(5 / 1,12 \mathrm{~mL})$ before the mixture was heated for $1 \mathrm{~h}$ at $75^{\circ} \mathrm{C}$. After cooling to room temperature, the flask was opened to air and the phosphonium chloride salt was extracted with $\mathrm{CHCl}_{3}(3 \times 40 \mathrm{~mL})$, washed with $\mathrm{H}_{2} \mathrm{O}(5 \times 20 \mathrm{~mL})$, dried over $\mathrm{MgSO}_{4}$, and concentrated in vacuo. The resulting oily orange residue was then dissolved in a minimum amount of $\mathrm{CH}_{2} \mathrm{Cl}_{2}$ and precipitated into pentane. The resulting yellow powder was isolated by centrifugation before it was again dissolved, precipitated, and filtered to yield $\mathbf{6 a}$ as an orange powder. Yield $=1.20$ g, 98\%. M.p. $74-76{ }^{\circ} \mathrm{C} .{ }^{1} \mathrm{H}$ NMR (DMSO- $\left.d_{6}\right): \delta 7.59$ $\left(\mathrm{d},{ }^{3} J_{\mathrm{HH}}=8 \mathrm{~Hz}, 2 \mathrm{H}\right.$, aryl $\left.\mathrm{CH}\right), 7.46\left(\mathrm{dd},{ }^{3} J_{\mathrm{HH}}=8 \mathrm{~Hz},{ }^{5} J_{\mathrm{HP}}=2 \mathrm{~Hz}\right.$, $2 \mathrm{H}$, aryl CH), $6.74\left(\mathrm{dd},{ }^{3} J_{\mathrm{HH}, \text { cis }}=11 \mathrm{~Hz},{ }^{3} J_{\mathrm{HH}, \text { trans }}=18 \mathrm{~Hz}, 1 \mathrm{H}\right.$, $\left.\operatorname{ArCH}=\mathrm{CH}_{2}\right), 5.86\left(\mathrm{~d},{ }^{3} J_{\mathrm{HH}, \text { trans }}=18 \mathrm{~Hz}, 1 \mathrm{H}, \mathrm{ArCH}=\mathrm{CH}_{2}\right), 5.26$ $\left(\mathrm{d},{ }^{3} J_{\mathrm{HH}, c i s}=11 \mathrm{~Hz}, 1 \mathrm{H}, \mathrm{ArCH}=\mathrm{CH}_{2}\right), 4.17$ (pseudo-t, ${ }^{3} J_{\mathrm{HH}}=$ $\left.2 \mathrm{~Hz}, 6 \mathrm{H}, \beta-\mathrm{C}_{5} \mathrm{H}_{4} \mathrm{R}\right), 4.12\left(\mathrm{~s}, 15 \mathrm{H}, \mathrm{C}_{5} H_{5}\right), 4.10$ (pseudo-t, ${ }^{3} J_{\mathrm{HH}}=2$ $\mathrm{Hz}, 6 \mathrm{H}, \alpha-\mathrm{C}_{5} H_{4} \mathrm{R}$ ), 4.06 (d, ${ }^{2} J_{\mathrm{HP}}=15 \mathrm{~Hz}, 2 \mathrm{H}, \mathrm{PCH} \mathrm{H}_{2} \mathrm{Ar}$ ), 2.59-2.43 (m, $12 \mathrm{H}, \mathrm{C}_{5} \mathrm{H}_{4} \mathrm{CH}_{2} \mathrm{CH}_{2}$ and $\mathrm{C}_{5} \mathrm{H}_{4} \mathrm{CH}_{2} \mathrm{CH}_{2}$, overlaps with residual $\mathrm{CD}_{3} \mathrm{CD}_{2} \mathrm{HSO}$ signal). ${ }^{13} \mathrm{C}\left\{{ }^{1} \mathrm{H}\right\}$ NMR (DMSO- $d_{6}$ ): $\delta 137.0$ $\left(\mathrm{d}, J_{\mathrm{CP}}=3 \mathrm{~Hz}\right), 135.9\left(\mathrm{~d}, J_{\mathrm{CP}}=2 \mathrm{~Hz}\right), 130.4\left(\mathrm{~d}, J_{\mathrm{CP}}=6 \mathrm{~Hz}\right), 128.8$ $\left(\mathrm{d}, J_{\mathrm{CP}}=8 \mathrm{~Hz}\right), 127.0\left(\mathrm{~d}, J_{\mathrm{CP}}=3 \mathrm{~Hz}\right), 115.1(\mathrm{~s}), 86.8\left(\mathrm{~d}, J_{\mathrm{CP}}=17\right.$ $\mathrm{Hz}$ ), 68.5 (s), 67.7 (s), 67.4 (s), 25.4 (d, $\left.J_{\mathrm{CP}}=43 \mathrm{~Hz}\right), 20.8$ (d, $J_{\mathrm{CP}}$ $=2 \mathrm{~Hz}), 19.3\left(\mathrm{~d}, J_{\mathrm{CP}}=44 \mathrm{~Hz}\right) \cdot{ }^{31} \mathrm{P}\left\{{ }^{1} \mathrm{H}\right\}$ NMR (DMSO- $\left.d_{6}\right): \delta 31.1$ (s). FT-IR: $3092(\mathrm{w}), 3009(\mathrm{w}), 2922(\mathrm{~m}), 2455(\mathrm{w}), 1629(\mathrm{w})$, $1511(\mathrm{w}), 1410(\mathrm{w}), 1219(\mathrm{w}), 1105(\mathrm{~m}), 1000(\mathrm{~m}), 922(\mathrm{w}), 820$ (m), $753(\mathrm{~s}), 660(\mathrm{w}) \mathrm{cm}^{-1}$. UV-vis (THF): $\lambda_{\max } 285 \mathrm{~nm}(\varepsilon=2620$ $\left.\mathrm{M}^{-1} \mathrm{~cm}^{-1}\right), 295 \mathrm{~nm}\left(\varepsilon=1380 \mathrm{M}^{-1} \mathrm{~cm}^{-1}\right), 325 \mathrm{~nm}\left(\varepsilon=290 \mathrm{M}^{-1}\right.$ $\left.\mathrm{cm}^{-1}\right), 436 \mathrm{~nm}\left(\varepsilon=300 \mathrm{M}^{-1} \mathrm{~cm}^{-1}\right)$. Mass Spec. (ESI, +ve mode): exact mass calculated for $\left[\mathrm{C}_{45} \mathrm{H}_{48}{ }^{56} \mathrm{Fe}_{3} \mathrm{P}\right]^{+}: 787.1542$; exact mass found: 787.1563; difference: $+2.7 \mathrm{ppm}$.

Phosphonium chloride salt $\mathbf{6 b}(2 \times$ Fc, $1 \times$ Rc). From tertiary phosphine $5 \mathbf{b}(1.00 \mathrm{~g}, 1.40 \mathrm{mmol})$ and 4-vinylbenzyl chloride (217 $\mu \mathrm{L}, 1.54 \mathrm{mmol}, 1.1$ equiv.). Yield $=1.16 \mathrm{~g}, 96 \%$. M.p. $80-82{ }^{\circ} \mathrm{C} .{ }^{1} \mathrm{H}$ NMR (DMSO- $\left.d_{6}\right): \delta 7.62\left(\mathrm{~d},{ }^{3} J_{\mathrm{HH}}=8 \mathrm{~Hz}, 2 \mathrm{H}\right.$, aryl $\mathrm{CH}), 7.48\left(\mathrm{dd},{ }^{3} \mathrm{~J}_{\mathrm{HH}}=7 \mathrm{~Hz},{ }^{5} J_{\mathrm{HP}}=2 \mathrm{~Hz}, 2 \mathrm{H}\right.$, aryl $\left.\mathrm{CH}\right)$, $6.78\left(\mathrm{dd},{ }^{3} J_{\mathrm{HH}, \text { cis }}=11 \mathrm{~Hz},{ }^{3} J_{\mathrm{HH}, \text { trans }}=18 \mathrm{~Hz}, 1 \mathrm{H}, \operatorname{ArCH}=\mathrm{CH}_{2}\right)$, $5.90\left(\mathrm{~d},{ }^{3} J_{\mathrm{HH}, \text { trans }}=18 \mathrm{~Hz}, 1 \mathrm{H}, \mathrm{ArCH}=\mathrm{CH}_{2}\right), 5.30\left(\mathrm{~d},{ }^{3} J_{\mathrm{HH}, c i s}=\right.$ $\left.11 \mathrm{~Hz}, 1 \mathrm{H}, \mathrm{ArCH}=\mathrm{CH}_{2}\right), 4.65\left[\mathrm{~s}, 2 \mathrm{H}, \beta-\mathrm{C}_{5} H_{4} \mathrm{R}(\mathrm{Ru})\right], 4.57[\mathrm{~s}, 5 \mathrm{H}$, $\left.\mathrm{C}_{5} H_{5}(\mathrm{Ru})\right], 4.51\left[\mathrm{~s}, 2 \mathrm{H}, \alpha-\mathrm{C}_{5} H_{5} \mathrm{R}(\mathrm{Ru})\right], 4.21\left[\mathrm{~s}, 4 \mathrm{H}, \beta-\mathrm{C}_{5} H_{4} \mathrm{R}(\mathrm{Fe})\right]$, $4.16\left[\mathrm{~s}, 10 \mathrm{H}, \mathrm{C}_{5} H_{5}(\mathrm{Fe})\right], 4.13\left[\mathrm{~s}, 4 \mathrm{H}, \alpha-\mathrm{C}_{5} H_{5} \mathrm{R}(\mathrm{Fe})\right], 4.10(\mathrm{~d}$, $\left.{ }^{2} J_{\mathrm{HP}}=16 \mathrm{~Hz}, 2 \mathrm{H}, \mathrm{PCH} \mathrm{H}_{2} \mathrm{Ar}\right), 2.65-2.30\left(\mathrm{~m}, 12 \mathrm{H}, \mathrm{C}_{5} \mathrm{H}_{4} \mathrm{CH}_{2} \mathrm{CH}_{2}\right.$ and $\mathrm{C}_{5} \mathrm{H}_{4} \mathrm{CH}_{2} \mathrm{CH}_{2}$, overlaps with residual $\mathrm{CD}_{3} \mathrm{CD}_{2} \mathrm{HSO}$ signal). ${ }^{13} \mathrm{C}\left\{{ }^{1} \mathrm{H}\right\}$ NMR (DMSO- $d_{6}$ ): $\delta 137.0\left(\mathrm{~d}, J_{\mathrm{CP}}=3 \mathrm{~Hz}\right.$ ), $135.9(\mathrm{~s})$, $130.4\left(\mathrm{~d}, J_{\mathrm{CP}}=5 \mathrm{~Hz}\right), 128.8\left(\mathrm{~d}, J_{\mathrm{CP}}=9 \mathrm{~Hz}\right), 126.9\left(\mathrm{~d}, J_{\mathrm{CP}}=3 \mathrm{~Hz}\right)$, $115.1(\mathrm{~s}), 91.2\left(\mathrm{~d}, J_{\mathrm{CP}}=18 \mathrm{~Hz}\right), 86.8\left(\mathrm{~d}, J_{\mathrm{CP}}=17 \mathrm{~Hz}\right), 70.6(\mathrm{~s})$, $70.4(\mathrm{~s}), 69.6$ (s), $68.4(\mathrm{~s}), 67.7(\mathrm{~s}), 67.3(\mathrm{~s}), 25.3$ (d, $\left.J_{\mathrm{CP}}=43 \mathrm{~Hz}\right)$, 20.8 (s), 20.2 (s), 20.0 (d, $\left.J_{\mathrm{CP}}=45 \mathrm{~Hz}\right), 19.3\left(\mathrm{~d}, J_{\mathrm{CP}}=45 \mathrm{~Hz}\right) \cdot{ }^{31} \mathrm{P}$ $\left\{{ }^{1} \mathrm{H}\right\}$ NMR (DMSO- $\left.d_{6}\right): \delta 30.9(\mathrm{~s})$. FT-IR: $3091(\mathrm{w}), 3008(\mathrm{w})$, $2922(\mathrm{~m}), 2455(\mathrm{w}), 1629(\mathrm{w}), 1511(\mathrm{w}), 1410(\mathrm{w}), 1221(\mathrm{w})$, $1104(\mathrm{~m}), 999(\mathrm{~m}), 918(\mathrm{w}), 811(\mathrm{~m}), 753(\mathrm{~s}), 661(\mathrm{w}) \mathrm{cm}^{-1}$. UV-vis (THF): $\lambda_{\max } 287 \mathrm{~nm}\left(\varepsilon=2170 \mathrm{M}^{-1} \mathrm{~cm}^{-1}\right), 295 \mathrm{~nm}(\varepsilon=$ $\left.1290 \mathrm{M}^{-1} \mathrm{~cm}^{-1}\right), 320 \mathrm{~nm}\left(\varepsilon=420 \mathrm{M}^{-1} \mathrm{~cm}^{-1}\right), 431 \mathrm{~nm}(\varepsilon=220$
$\mathrm{M}^{-1} \mathrm{~cm}^{-1}$ ). Mass Spec. (ESI, +ve mode): exact mass calculated for $\left[\mathrm{C}_{45} \mathrm{H}_{48}{ }^{56} \mathrm{Fe}_{2} \mathrm{P}^{102} \mathrm{Ru}\right]^{+}:$833.1236; exact mass found: 833.1259; difference: +2.8 ppm.

Phosphonium chloride salt $\mathbf{6 c}(\mathbf{1} \times \mathrm{Fc}, \mathbf{2} \times \mathbf{R c})$. From tertiary phosphine 5c (1.00 g, $1.32 \mathrm{mmol})$ and 4-vinylbenzyl chloride (204 $\mu \mathrm{L}, 1.45 \mathrm{mmol}, 1.1$ equiv.). Yield $=1.14 \mathrm{~g}, 95 \%$. M.p. $84-86{ }^{\circ} \mathrm{C} .{ }^{1} \mathrm{H}$ NMR (DMSO- $\left.d_{6}\right): \delta 7.62\left(\mathrm{~d},{ }^{3} J_{\mathrm{HH}}=8 \mathrm{~Hz}, 2 \mathrm{H}\right.$, aryl $\mathrm{CH}$ ), $7.44\left(\mathrm{dd},{ }^{3} J_{\mathrm{HH}}=7 \mathrm{~Hz},{ }^{5} J_{\mathrm{HP}}=2 \mathrm{~Hz}, 2 \mathrm{H}\right.$, aryl $\left.\mathrm{CH}\right), 6.78$ $\left(\mathrm{dd},{ }^{3} J_{\mathrm{HH}, \text { cis }}=11 \mathrm{~Hz},{ }^{3} J_{\mathrm{HH}, \text { trans }}=18 \mathrm{~Hz}, 1 \mathrm{H}, \mathrm{ArCH}=\mathrm{CH}_{2}\right), 5.92$ $\left(\mathrm{d},{ }^{3} J_{\mathrm{HH}, \text { trans }}=18 \mathrm{~Hz}, 1 \mathrm{H}, \mathrm{ArCH}=\mathrm{CH}_{2}\right), 5.31\left(\mathrm{~d},{ }^{3} J_{\mathrm{HH}, \text { cis }}=11 \mathrm{~Hz}\right.$, $\left.1 \mathrm{H}, \mathrm{ArCH}=\mathrm{CH}_{2}\right), 4.65\left[\mathrm{~s}, 4 \mathrm{H}, \beta-\mathrm{C}_{5} H_{4} \mathrm{R}(\mathrm{Ru})\right], 4.56[\mathrm{~s}, 10 \mathrm{H}$, $\left.\mathrm{C}_{5} H_{5}(\mathrm{Ru})\right], 4.52\left[\mathrm{~s}, 4 \mathrm{H}, \alpha-\mathrm{C}_{5} H_{5} \mathrm{R}(\mathrm{Ru})\right], 4.21$ [pseudo-t, ${ }^{3} J_{\mathrm{HH}}=$ $\left.2 \mathrm{~Hz}, 2 \mathrm{H}, \beta-\mathrm{C}_{5} H_{4} \mathrm{R}(\mathrm{Fe})\right], 4.16\left[\mathrm{~s}, 5 \mathrm{H}, \mathrm{C}_{5} H_{5}(\mathrm{Fe})\right], 4.14$ [pseudo-t, $\left.{ }^{3} J_{\mathrm{HH}}=2 \mathrm{~Hz}, 2 \mathrm{H}, \alpha-\mathrm{C}_{5} H_{5} \mathrm{R}(\mathrm{Fe})\right], 4.06\left(\mathrm{~d},{ }^{2} J_{\mathrm{HP}}=16 \mathrm{~Hz}, 2 \mathrm{H}\right.$, $\left.\mathrm{PCH}_{2} \mathrm{Ar}\right), 2.60-2.30\left(\mathrm{~m}, 12 \mathrm{H}, \mathrm{C}_{5} \mathrm{H}_{4} \mathrm{CH}_{2} \mathrm{CH}_{2}\right.$ and $\mathrm{C}_{5} \mathrm{H}_{4} \mathrm{CH}_{2} \mathrm{CH}_{2}$, overlaps with residual $\mathrm{CD}_{3} \mathrm{CD}_{2} \mathrm{HSO}$ signal). ${ }^{13} \mathrm{C}\left\{{ }^{1} \mathrm{H}\right\}$ NMR $\left(\mathrm{DMSO}-d_{6}\right): \delta 137.0\left(\mathrm{~d}, J_{\mathrm{CP}}=5 \mathrm{~Hz}\right), 135.9(\mathrm{~s}), 130.3\left(\mathrm{~d}, J_{\mathrm{CP}}=\right.$ $5 \mathrm{~Hz}), 128.7\left(\mathrm{~d}, J_{\mathrm{CP}}=9 \mathrm{~Hz}\right), 126.9\left(\mathrm{~d}, J_{\mathrm{CP}}=3 \mathrm{~Hz}\right), 115.1(\mathrm{~s}), 91.1$ $\left(\mathrm{d}, J_{\mathrm{CP}}=17 \mathrm{~Hz}\right), 86.7\left(\mathrm{~d}, J_{\mathrm{CP}}=17 \mathrm{~Hz}\right), 70.6(\mathrm{~s}), 70.4(\mathrm{~s}), 69.7(\mathrm{~s})$, $68.4(\mathrm{~s}), 67.7(\mathrm{~s}), 67.4(\mathrm{~s}), 25.3\left(\mathrm{~d}, J_{\mathrm{CP}}=43 \mathrm{~Hz}\right), 20.8(\mathrm{~s}), 20.3(\mathrm{~d}$, $\left.J_{\mathrm{CP}}=3 \mathrm{~Hz}\right), 20.0\left(\mathrm{~d}, J_{\mathrm{CP}}=44 \mathrm{~Hz}\right), 19.3\left(\mathrm{~d}, J_{\mathrm{CP}}=45 \mathrm{~Hz}\right) \cdot{ }^{31} \mathrm{P}\left\{{ }^{1} \mathrm{H}\right\}$ NMR (DMSO- $\left.d_{6}\right): \delta 30.8$ (s). FT-IR: $3095(\mathrm{w}), 3010(\mathrm{w}), 2923(\mathrm{~m})$, $2455(\mathrm{w}), 1630(\mathrm{w}), 1511(\mathrm{w}), 1409(\mathrm{w}), 1219(\mathrm{w}), 1101(\mathrm{~m})$,

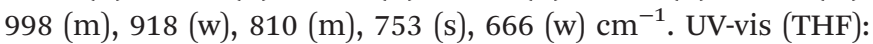
$\lambda_{\text {max }} 287 \mathrm{~nm}\left(\varepsilon=2520 \mathrm{M}^{-1} \mathrm{~cm}^{-1}\right), 295 \mathrm{~nm}\left(\varepsilon=1600 \mathrm{M}^{-1} \mathrm{~cm}^{-1}\right)$, $320 \mathrm{~nm}\left(\varepsilon=680 \mathrm{M}^{-1} \mathrm{~cm}^{-1}\right), 434 \mathrm{~nm}\left(\varepsilon=150 \mathrm{M}^{-1} \mathrm{~cm}^{-1}\right)$. Mass Spec. (ESI, +ve mode): exact mass calculated for $\left[\mathrm{C}_{45} \mathrm{H}_{48}{ }^{56} \mathrm{FeP}^{96} \mathrm{Ru}^{99} \mathrm{Ru}\right]^{+}$: 870.0978; exact mass found: 870.1002; difference: +2.8 ppm.

Phosphonium chloride salt $\mathbf{6 d}(3 \times \mathbf{R c})$. From tertiary phosphine 5d (1.00 g, $1.24 \mathrm{mmol})$ and 4-vinylbenzyl chloride (192 $\mu \mathrm{L}, 1.36 \mathrm{mmol}, 1.1$ eq.). Yield $=1.12 \mathrm{~g}, 94 \%$. M.p. $102-104{ }^{\circ} \mathrm{C} .{ }^{1} \mathrm{H}$ NMR (DMSO- $\left.d_{6}\right): \delta 7.62\left(\mathrm{~d},{ }^{3} J_{\mathrm{HH}}=8 \mathrm{~Hz}\right.$, $2 \mathrm{H}$, aryl $\mathrm{CH}), 7.40\left(\mathrm{dd},{ }^{3} J_{\mathrm{HH}}=8 \mathrm{~Hz},{ }^{5} J_{\mathrm{HP}}=2 \mathrm{~Hz}, 2 \mathrm{H}\right.$, aryl $\mathrm{CH}), 6.77\left(\mathrm{dd},{ }^{3} J_{\mathrm{HH}, \text { cis }}=11 \mathrm{~Hz},{ }^{3} J_{\mathrm{HH}, \text { trans }}=18 \mathrm{~Hz}, 1 \mathrm{H}\right.$, $\left.\mathrm{ArCH}=\mathrm{CH}_{2}\right), 5.90\left(\mathrm{~d},{ }^{3} J_{\mathrm{HH}, \text { trans }}=18 \mathrm{~Hz}, 1 \mathrm{H}, \mathrm{ArCH}=\mathrm{CH}_{2}\right), 5.31$ $\left(\mathrm{d},{ }^{3} J_{\mathrm{HH}, c i s}=11 \mathrm{~Hz}, 1 \mathrm{H}, \mathrm{ArCH}=\mathrm{CH}_{2}\right), 4.64$ (pseudo-t, ${ }^{3} J_{\mathrm{HH}}=$ $2 \mathrm{~Hz}, 6 \mathrm{H}, \beta-\mathrm{C}_{5} \mathrm{H}_{4} \mathrm{R}$ ), $4.55\left(\mathrm{~s}, 15 \mathrm{H}, \mathrm{C}_{5} H_{5}\right), 4.51$ (pseudo-t, ${ }^{3} \mathrm{~J}_{\mathrm{HH}}=2$ $\mathrm{Hz}, 6 \mathrm{H}, \alpha-\mathrm{C}_{5} \mathrm{H}_{4} \mathrm{R}$ ), $3.98\left(\mathrm{~d},{ }^{2} J_{\mathrm{HP}}=15 \mathrm{~Hz}, 2 \mathrm{H}, \mathrm{PCH}_{2} \mathrm{Ar}\right.$ ), 2.27-2.47 $\left(\mathrm{m}, \quad 12 \mathrm{H}, \mathrm{C}_{5} \mathrm{H}_{4} \mathrm{CH}_{2} \mathrm{CH}_{2}\right.$ and $\left.\mathrm{C}_{5} \mathrm{H}_{4} \mathrm{CH}_{2} \mathrm{CH}_{2}\right) .{ }^{13} \mathrm{C}\left\{{ }^{1} \mathrm{H}\right\} \quad \mathrm{NMR}$ $\left(\mathrm{DMSO}-d_{6}\right): \delta 137.1\left(\mathrm{~d}, J_{\mathrm{CP}}=3 \mathrm{~Hz}\right), 135.9(\mathrm{~s}), 130.3\left(\mathrm{~d}, J_{\mathrm{CP}}=5\right.$ $\mathrm{Hz}), 128.6\left(\mathrm{~d}, J_{\mathrm{CP}}=9 \mathrm{~Hz}\right), 127.0(\mathrm{~s}), 115.3(\mathrm{~s}), 91.1$ (d, $J_{\mathrm{CP}}=18$ $\mathrm{Hz}$ ), 70.6 (s), 70.5 (s), 69.8 (s), 25.4 (d, $J_{\mathrm{CP}}=44 \mathrm{~Hz}$ ), 20.4 (s), $20.0\left(\mathrm{~d}, J_{\mathrm{CP}}=45 \mathrm{~Hz}\right) .{ }^{31} \mathrm{P}\left\{{ }^{1} \mathrm{H}\right\}$ NMR (DMSO- $\left.d_{6}\right): \delta 30.8$ (s). FT-IR: 3093 (w), 3010 (w), 2919 (m), 2460 (w), 1670 (w), $1511(\mathrm{w}), 1409$ (m), $1218(\mathrm{w}), 1100(\mathrm{~m}), 997(\mathrm{~m}), 917$ (w), $809(\mathrm{~s}), 752(\mathrm{~s}), 660$ (w) $\mathrm{cm}^{-1}$. UV-vis (THF): $\lambda_{\max } 287 \mathrm{~nm}\left(\varepsilon=2060 \mathrm{M}^{-1} \mathrm{~cm}^{-1}\right)$, $297 \mathrm{~nm}\left(\varepsilon=1560 \mathrm{M}^{-1} \mathrm{~cm}^{-1}\right), 315 \mathrm{~nm}\left(\varepsilon=890 \mathrm{M}^{-1} \mathrm{~cm}^{-1}\right)$. Mass Spec. (ESI, +ve mode): exact mass calculated for $\left[\mathrm{C}_{45} \mathrm{H}_{48}{ }^{96} \mathrm{Ru}^{99} \mathrm{Ru}^{100} \mathrm{RuP}\right]^{+}$: 914.0671; exact mass found: 914.0678; difference: +0.8 ppm.

Representative procedure for the preparation of phosphonium salts $7 \mathbf{a}-\mathbf{d}$

Phosphonium triflate salt $7 \mathbf{a}(3 \times$ Fc). In a Schlenk flask, $6 a$ $(1.00 \mathrm{~g}, 1.22 \mathrm{mmol})$ was dissolved in dry and degassed $\mathrm{CHCl}_{3}$ 
(10 mL) before NaOTf ( $0.63 \mathrm{~g}, 3.6 \mathrm{mmol}, 3$ equiv.) was charged into the reaction flask. The resulting mixture was stirred at $20{ }^{\circ} \mathrm{C}$ for $16 \mathrm{~h}$, gravity filtered to remove precipitate, washed with $\mathrm{H}_{2} \mathrm{O}(3 \times 10 \mathrm{~mL})$, dried over $\mathrm{MgSO}_{4}$, and gravity filtered. NaOTf (0.21 g, $1.2 \mathrm{mmol}, 1$ equiv.) was once again charged into a flask containing the filtrate before the contents were stirred for an additional $16 \mathrm{~h}$. The reaction mixture was then gravity filtered to remove precipitate, washed with $\mathrm{H}_{2} \mathrm{O}(3 \times$ $10 \mathrm{~mL}$ ), dried over $\mathrm{MgSO}_{4}$, and gravity filtered before it was concentrated in vacuo. The resulting orange residue was then dried in vacuo for $2 \mathrm{~h}$ at $45{ }^{\circ} \mathrm{C}$ to remove residual solvent to afford monomer 7a as an orange solid. Yield $=1.12 \mathrm{~g}, 98 \%$. $\mathrm{M}$. p. $66-68{ }^{\circ} \mathrm{C} .{ }^{1} \mathrm{H}$ NMR (DMSO- $\left.d_{6}\right): \delta 7.61\left(\mathrm{~d},{ }^{3} J_{\mathrm{HH}}=8 \mathrm{~Hz}, 2 \mathrm{H}\right.$, aryl $\mathrm{CH}), 7.44\left(\mathrm{dd},{ }^{3} J_{\mathrm{HH}}=8 \mathrm{~Hz},{ }^{5} J_{\mathrm{HP}}=2 \mathrm{~Hz}, 2 \mathrm{H}\right.$, aryl $\left.\mathrm{CH}\right), 6.76$ $\left(\mathrm{dd},{ }^{3} J_{\mathrm{HH}, \text { cis }}=11 \mathrm{~Hz},{ }^{3} J_{\mathrm{HH}, \text { trans }}=18 \mathrm{~Hz}, 1 \mathrm{H}, \mathrm{ArCH}=\mathrm{CH}_{2}\right), 5.89$ $\left(\mathrm{d},{ }^{3} \mathrm{~J}_{\mathrm{HH}, \text { trans }}=18 \mathrm{~Hz}, 1 \mathrm{H}, \mathrm{ArCH}=\mathrm{CH}_{2}\right), 5.29\left(\mathrm{~d},{ }^{3} J_{\mathrm{HH}, \text { cis }}=11 \mathrm{~Hz}\right.$, $\left.1 \mathrm{H}, \mathrm{ArCH}=\mathrm{CH}_{2}\right), 4.19\left(\mathrm{~s}, 6 \mathrm{H}, \beta-\mathrm{C}_{5} H_{4} \mathrm{R}\right), 4.14\left(\mathrm{~s}, 15 \mathrm{H}, \mathrm{C}_{5} H_{5}\right)$, $4.13\left(\mathrm{~s}, 6 \mathrm{H}, \alpha-\mathrm{C}_{5} \mathrm{H}_{4} \mathrm{R}\right), 3.96\left(\mathrm{~d},{ }^{2} J_{\mathrm{HP}}=15 \mathrm{~Hz}, 2 \mathrm{H}, \mathrm{PCH}_{2} \mathrm{Ar}\right)$, 2.60-2.42 (m, 12H, $\mathrm{C}_{5} \mathrm{H}_{4} \mathrm{CH}_{2} \mathrm{CH}_{2}$ and $\mathrm{C}_{5} \mathrm{H}_{4} \mathrm{CH}_{2} \mathrm{CH}_{2}$, overlaps with residual $\mathrm{CD}_{3} \mathrm{CD}_{2} \mathrm{HSO}$ signal). ${ }^{13} \mathrm{C}\left\{{ }^{1} \mathrm{H}\right\}$ NMR (DMSO- $d_{6}$ ): $\delta$ $137.1\left(\mathrm{~d}, J_{\mathrm{CP}}=5 \mathrm{~Hz}\right), 135.9(\mathrm{~s}), 130.4\left(\mathrm{~d}, J_{\mathrm{CP}}=3 \mathrm{~Hz}\right), 128.6(\mathrm{~d}$, $\left.J_{\mathrm{CP}}=9 \mathrm{~Hz}\right), 127.0(\mathrm{~s}), 120.7\left(\mathrm{q}, J_{\mathrm{CF}}=322 \mathrm{~Hz}\right), 115.2(\mathrm{~s}), 86.8(\mathrm{~d}$, $\left.J_{\mathrm{CP}}=18 \mathrm{~Hz}\right), 68.4(\mathrm{~s}), 67.7(\mathrm{~s}), 67.4(\mathrm{~s}), 25.4\left(\mathrm{~d}, J_{\mathrm{CP}}=44 \mathrm{~Hz}\right), 20.8$ $(\mathrm{s}), 19.2\left(\mathrm{~d}, J_{\mathrm{CP}}=45 \mathrm{~Hz}\right) \cdot{ }^{19} \mathrm{~F}$ NMR (DMSO- $\left.d_{6}\right): \delta-77.6(\mathrm{~s}) \cdot{ }^{31} \mathrm{P}$ $\left\{{ }^{1} \mathrm{H}\right\}$ NMR (DMSO- $\left.d_{6}\right): \delta 31.2(\mathrm{~s})$. FT-IR: $3095(\mathrm{w}), 3010(\mathrm{w}), 2913$ (w), 1512 (w), 1410 (w), 1262 (s), 1157 (m), $1124(\mathrm{w}), 1105$ (w), $1030(\mathrm{~s}), 1000(\mathrm{w}), 922(\mathrm{w}), 821(\mathrm{~m}), 754(\mathrm{~m}), 637$ (s) cm $\mathrm{cm}^{-1}$. UV-vis (THF): $\lambda_{\max } 285 \mathrm{~nm}\left(\varepsilon=2470 \mathrm{M}^{-1} \mathrm{~cm}^{-1}\right), 295 \mathrm{~nm}\left(\varepsilon=1290 \mathrm{M}^{-1}\right.$ $\left.\mathrm{cm}^{-1}\right), 325 \mathrm{~nm}\left(\varepsilon=260 \mathrm{M}^{-1} \mathrm{~cm}^{-1}\right), 436 \mathrm{~nm}\left(\varepsilon=320 \mathrm{M}^{-1} \mathrm{~cm}^{-1}\right)$. Mass Spec. (ESI, +ve mode): exact mass calculated for $\left[\mathrm{C}_{45} \mathrm{H}_{48}{ }^{56} \mathrm{Fe}_{3} \mathrm{P}\right]^{+}$: 787.1542; exact mass found: 787.1564; difference: +2.7 ppm. Anal. Calcd (\%) for $\mathrm{C}_{46} \mathrm{H}_{48} \mathrm{~F}_{3} \mathrm{Fe}_{3} \mathrm{O}_{3} \mathrm{PS}$ : C, 59.00; H, 5.17. Found: C, 58.94; H, 5.37.

Phosphonium triflate salt $7 \mathbf{b}(2 \times$ Fc, $1 \times$ Rc $)$. From phosphonium chloride $6 \mathbf{b}$ (1.00 g, $1.15 \mathrm{mmol})$ and NaOTf (0.60 g, $3.5 \mathrm{mmol}, 3$ equiv. for the first metathesis reaction and $0.20 \mathrm{~g}$, $1.2 \mathrm{mmol}, 1$ equiv. for the second metathesis reaction). Yield $=$ 1.11 g, 98\%. M.p. $68-70{ }^{\circ} \mathrm{C} .{ }^{1} \mathrm{H}$ NMR (DMSO- $d_{6}$ ): $\delta 7.64$ (d, ${ }^{3} J_{\mathrm{HH}}=8 \mathrm{~Hz}, 2 \mathrm{H}$, aryl $\left.\mathrm{CH}\right), 7.44\left(\mathrm{~d},{ }^{3} J_{\mathrm{HH}}=7 \mathrm{~Hz}, 2 \mathrm{H}\right.$, aryl $\left.\mathrm{CH}\right)$, $6.78\left(\mathrm{dd},{ }^{3} \mathrm{~J}_{\mathrm{HH}, \text { cis }}=11 \mathrm{~Hz},{ }^{3} J_{\mathrm{HH}, \text { trans }}=18 \mathrm{~Hz}, 1 \mathrm{H}, \operatorname{ArCH}=\mathrm{CH}_{2}\right)$, $5.91\left(\mathrm{~d},{ }^{3} J_{\mathrm{HH}, \text { trans }}=18 \mathrm{~Hz}, 1 \mathrm{H}, \mathrm{ArCH}=\mathrm{CH}_{2}\right), 5.31\left(\mathrm{~d},{ }^{3} J_{\mathrm{HH}, \text { cis }}=\right.$ $\left.11 \mathrm{~Hz}, 1 \mathrm{H}, \mathrm{ArCH}=\mathrm{CH}_{2}\right), 4.64\left[\mathrm{~s}, 2 \mathrm{H}, \beta-\mathrm{C}_{5} H_{4} \mathrm{R}(\mathrm{Ru})\right], 4.56[\mathrm{~s}, 5 \mathrm{H}$, $\left.\mathrm{C}_{5} H_{5}(\mathrm{Ru})\right], 4.52\left[\mathrm{~s}, 2 \mathrm{H}, \alpha-\mathrm{C}_{5} H_{5} \mathrm{R}(\mathrm{Ru})\right], 4.20\left[\mathrm{~s}, 4 \mathrm{H}, \beta-\mathrm{C}_{5} H_{4} \mathrm{R}(\mathrm{Fe})\right]$, $4.15\left[\mathrm{~s}, 10 \mathrm{H}, \mathrm{C}_{5} H_{5}(\mathrm{Fe})\right], 4.14\left[\mathrm{~s}, 4 \mathrm{H}, \alpha-\mathrm{C}_{5} H_{5} \mathrm{R}(\mathrm{Fe})\right], 3.96(\mathrm{~d}$, $\left.{ }^{2} J_{\mathrm{HP}}=15 \mathrm{~Hz}, 2 \mathrm{H}, \mathrm{PCH} \mathrm{H}_{2} \mathrm{Ar}\right), 2.57-2.32\left(\mathrm{~m}, 12 \mathrm{H}, \mathrm{C}_{5} \mathrm{H}_{4} \mathrm{CH}_{2} \mathrm{CH}_{2}\right.$ and $\mathrm{C}_{5} \mathrm{H}_{4} \mathrm{CH}_{2} \mathrm{CH}_{2}$, overlaps with residual $\mathrm{CD}_{3} \mathrm{CD}_{2} \mathrm{HSO}$ signal). ${ }^{13} \mathrm{C}\left\{{ }^{1} \mathrm{H}\right\}$ NMR (DMSO- $d_{6}$ ): $\delta 137.2\left(\mathrm{~d}, J_{\mathrm{CP}}=3 \mathrm{~Hz}\right.$ ), $135.9(\mathrm{~s})$, $130.4\left(\mathrm{~d}, J_{\mathrm{CP}}=6 \mathrm{~Hz}\right), 128.6\left(\mathrm{~d}, J_{\mathrm{CP}}=9 \mathrm{~Hz}\right), 127.1\left(\mathrm{~d}, J_{\mathrm{CP}}=2 \mathrm{~Hz}\right)$, $120.7\left(\mathrm{q}, J_{\mathrm{CF}}=322 \mathrm{~Hz}\right), 115.3\left(\mathrm{~d}, J_{\mathrm{CP}}=164 \mathrm{~Hz}\right), 91.2\left(\mathrm{~d}, J_{\mathrm{CP}}=\right.$ $18 \mathrm{~Hz}), 86.8\left(\mathrm{~d}, J_{\mathrm{CP}}=17 \mathrm{~Hz}\right), 70.6(\mathrm{~s}), 70.5(\mathrm{~s}), 69.8(\mathrm{~s}), 68.5(\mathrm{~s})$, $67.8(\mathrm{~s}), 67.5(\mathrm{~s}), 25.4\left(\mathrm{~d}, J_{\mathrm{CP}}=43 \mathrm{~Hz}\right), 20.9(\mathrm{~s}), 20.3(\mathrm{~s}), 20.1(\mathrm{~d}$, $\left.J_{\mathrm{CP}}=45 \mathrm{~Hz}\right), 19.3\left(\mathrm{~d}, J_{\mathrm{CP}}=45 \mathrm{~Hz}\right) .{ }^{19} \mathrm{~F} \mathrm{NMR}\left(\mathrm{DMSO}-d_{6}\right): \delta-77.8$ (s). ${ }^{31} \mathrm{P}\left\{{ }^{1} \mathrm{H}\right\}$ NMR (DMSO- $d_{6}$ ): $\delta 31.0$ (s). FT-IR: $3089(\mathrm{w}), 3011$ (w), $2912(\mathrm{w}), 1630(\mathrm{w}), 1512(\mathrm{w}), 1410(\mathrm{w}), 1262(\mathrm{~s}), 1224(\mathrm{~m})$, $1158(\mathrm{~m}), 1104(\mathrm{w}), 1030(\mathrm{~s}), 999(\mathrm{w}), 919(\mathrm{w}), 813(\mathrm{~m}), 755(\mathrm{~s})$, 637 (s) $\mathrm{cm}^{-1}$. UV-vis (THF): $\lambda_{\max } 284 \mathrm{~nm}\left(\varepsilon=2860 \mathrm{M}^{-1} \mathrm{~cm}^{-1}\right)$,
$295 \mathrm{~nm}\left(\varepsilon=1390 \mathrm{M}^{-1} \mathrm{~cm}^{-1}\right), 320 \mathrm{~nm}\left(\varepsilon=440 \mathrm{M}^{-1} \mathrm{~cm}^{-1}\right)$, $431 \mathrm{~nm}\left(\varepsilon=240 \mathrm{M}^{-1} \mathrm{~cm}^{-1}\right)$. Mass Spec. (ESI, +ve mode): exact mass calculated for $\left[\mathrm{C}_{45} \mathrm{H}_{48}{ }^{56} \mathrm{Fe}_{2} \mathrm{P}^{96} \mathrm{Ru}\right]^{+}$: 827.1268; exact mass found: 827.1274; difference: $+0.7 \mathrm{ppm}$. Anal. Calcd (\%) for $\mathrm{C}_{46} \mathrm{H}_{48} \mathrm{~F}_{3} \mathrm{Fe}_{2} \mathrm{O}_{3}$ PRuS: C, 56.28; H, 4.93. Found: C, 56.22; $\mathrm{H}$, 5.11 .

Phosphonium triflate salt 7c $(1 \times$ Fc, $2 \times$ Rc $)$. From phosphonium chloride $6 \mathrm{c}(1.00 \mathrm{~g}, 1.10 \mathrm{mmol})$ and NaOTf $(0.57 \mathrm{~g}$, $3.3 \mathrm{mmol}, 3$ equiv. for the first metathesis reaction and $0.19 \mathrm{~g}$, $1.1 \mathrm{mmol}, 1$ equiv. for the second metathesis reaction). Yield $=$ 1.09 g, 97\%. M.p. 70-72 ${ }^{\circ} \mathrm{C} .{ }^{1} \mathrm{H}$ NMR (DMSO- $d_{6}$ ): $\delta 7.64$ (d, ${ }^{3} J_{\mathrm{HH}}=8 \mathrm{~Hz}, 2 \mathrm{H}$, aryl $\left.\mathrm{CH}\right), 7.42\left(\mathrm{~d},{ }^{3} J_{\mathrm{HH}}=8 \mathrm{~Hz}, 2 \mathrm{H}\right.$, aryl $\left.\mathrm{CH}\right)$, $6.78\left(\mathrm{dd},{ }^{3} J_{\mathrm{HH}, \text { cis }}=11 \mathrm{~Hz},{ }^{3} J_{\mathrm{HH}, \text { trans }}=18 \mathrm{~Hz}, 1 \mathrm{H}, \mathrm{ArCH}=\mathrm{CH}_{2}\right)$, $5.92\left(\mathrm{~d},{ }^{3} J_{\mathrm{HH}, \text { trans }}=18 \mathrm{~Hz}, 1 \mathrm{H}, \mathrm{ArCH}=\mathrm{CH}_{2}\right), 5.32\left(\mathrm{~d},{ }^{3} J_{\mathrm{HH}, \text { cis }}=\right.$ $\left.11 \mathrm{~Hz}, 1 \mathrm{H}, \quad \mathrm{ArCH}=\mathrm{CH}_{2}\right), 4.65\left[\mathrm{~s}, 4 \mathrm{H}, \quad \beta-\mathrm{C}_{5} H_{4} \mathrm{R}(\mathrm{Ru})\right], 4.56$ $\left[\mathrm{s}, 10 \mathrm{H}, \mathrm{C}_{5} H_{5}(\mathrm{Ru})\right], 4.52\left[\mathrm{~s}, 4 \mathrm{H}, \alpha-\mathrm{C}_{5} H_{5} \mathrm{R}(\mathrm{Ru})\right], 4.21[\mathrm{~s}, 2 \mathrm{H}$, $\left.\beta-\mathrm{C}_{5} H_{4} \mathrm{R}(\mathrm{Fe})\right], 4.16\left[\mathrm{~s}, 5 \mathrm{H}, \mathrm{C}_{5} H_{5}(\mathrm{Fe})\right], 4.15\left[\mathrm{~s}, 2 \mathrm{H}, \alpha-\mathrm{C}_{5} H_{5} \mathrm{R}(\mathrm{Fe})\right]$, $3.96\left(\mathrm{~d},{ }^{2} J_{\mathrm{HP}}=15 \mathrm{~Hz}, 2 \mathrm{H}, \mathrm{PCH}_{2} \mathrm{Ar}\right), 2.57-2.21(\mathrm{~m}, 12 \mathrm{H}$, $\mathrm{C}_{5} \mathrm{H}_{4} \mathrm{CH}_{2} \mathrm{CH}_{2}$ and $\mathrm{C}_{5} \mathrm{H}_{4} \mathrm{CH}_{2} \mathrm{CH}_{2}$, overlaps with residual $\mathrm{CD}_{3} \mathrm{CD}_{2} \mathrm{HSO}$ signal). ${ }^{13} \mathrm{C}\left\{{ }^{1} \mathrm{H}\right\}$ NMR (DMSO- $\left.d_{6}\right): \delta 137.1\left(\mathrm{~d}, J_{\mathrm{CP}}=\right.$ $3 \mathrm{~Hz}), 135.9$ (s), 130.3 (d, $\left.J_{\mathrm{CP}}=5 \mathrm{~Hz}\right), 128.5$ (d, $\left.J_{\mathrm{CP}}=8 \mathrm{~Hz}\right)$, $127.0\left(\mathrm{~d}, J_{\mathrm{CP}}=2 \mathrm{~Hz}\right), 115.2(\mathrm{~s}), 91.0\left(\mathrm{~d}, J_{\mathrm{CP}}=18 \mathrm{~Hz}\right), 86.7(\mathrm{~d}$, $\left.J_{\mathrm{CP}}=17 \mathrm{~Hz}\right), 70.6(\mathrm{~s}), 70.4(\mathrm{~s}), 69.7(\mathrm{~s}), 68.4(\mathrm{~s}), 67.6(\mathrm{~s}), 67.4(\mathrm{~s})$, $25.3\left(\mathrm{~d}, J_{\mathrm{CP}}=44 \mathrm{~Hz}\right), 20.8(\mathrm{~s}), 20.2(\mathrm{~s}), 19.9\left(\mathrm{~d}, J_{\mathrm{CP}}=45 \mathrm{~Hz}\right), 19.2$ $\left(\mathrm{d}, J_{\mathrm{CP}}=45 \mathrm{~Hz}\right) \cdot{ }^{19} \mathrm{~F}$ NMR (DMSO- $\left.d_{6}\right): \delta-77.7(\mathrm{~s}) \cdot{ }^{31} \mathrm{P}\left\{{ }^{1} \mathrm{H}\right\}$ NMR (DMSO- $d_{6}$ ): $\delta 30.9$ (s). FT-IR: $3093(\mathrm{w}), 3013(\mathrm{w}), 2911(\mathrm{w}), 1630$ (w), 1512 (w), 1410 (w), 1262 (s), 1224 (s), 1159 (s), 1101 (m), 1030 (s), 998 (w), 918 (w), 811 (m), 756 (s), 637 (s) cm cm $^{-1}$ UV-vis (THF): $\lambda_{\max } 283 \mathrm{~nm}\left(\varepsilon=3260 \mathrm{M}^{-1} \mathrm{~cm}^{-1}\right), 295 \mathrm{~nm}(\varepsilon=1770$ $\left.\mathrm{M}^{-1} \mathrm{~cm}^{-1}\right), 320 \mathrm{~nm}\left(\varepsilon=730 \mathrm{M}^{-1} \mathrm{~cm}^{-1}\right), 434 \mathrm{~nm}(\varepsilon=140$ $\mathrm{M}^{-1} \mathrm{~cm}^{-1}$ ). Mass Spec. (ESI, +ve mode): exact mass calculated for $\left[\mathrm{C}_{45} \mathrm{H}_{48}{ }^{56} \mathrm{FeP}^{96} \mathrm{Ru}^{99} \mathrm{Ru}\right]^{+}$: 870.0978; exact mass found: 870.1000; difference: +2.5 ppm. Anal. Calcd (\%) for $\mathrm{C}_{46} \mathrm{H}_{48} \mathrm{~F}_{3} \mathrm{FeO}_{3} \mathrm{PRu}_{2} \mathrm{~S}$ : C, 53.80; H, 4.71. Found: C, 53.94; H, 4.77.

Phosphonium triflate salt $\mathbf{7 d}(\mathbf{3} \times \mathbf{R c})$. From phosphonium chloride 6d (1.00 g, $1.04 \mathrm{mmol})$ and NaOTf (0.54 g, $3.1 \mathrm{mmol}$, 3 equiv. for the first metathesis reaction and $0.18 \mathrm{~g}, 1.1 \mathrm{mmol}$, 1 equiv. for the second metathesis reaction). Yield $=1.07 \mathrm{~g}$, 96\%. M.p. $88-90{ }^{\circ} \mathrm{C} .{ }^{1} \mathrm{H}$ NMR (DMSO- $\left.d_{6}\right): \delta 7.62\left(\mathrm{~d},{ }^{3} J_{\mathrm{HH}}=\right.$ $8 \mathrm{~Hz}, 2 \mathrm{H}$, aryl CH), $7.38\left(\mathrm{dd},{ }^{3} J_{\mathrm{HH}}=8 \mathrm{~Hz},{ }^{5} J_{\mathrm{HP}}=2 \mathrm{~Hz}, 2 \mathrm{H}\right.$, aryl $\mathrm{CH}), 6.78\left(\mathrm{dd},{ }^{3} J_{\mathrm{HH}, \text { cis }}=11 \mathrm{~Hz},{ }^{3} J_{\mathrm{HH}, \text { trans }}=18 \mathrm{~Hz}, 1 \mathrm{H}\right.$, $\left.\operatorname{ArCH}=\mathrm{CH}_{2}\right), 5.92\left(\mathrm{~d},{ }^{3} J_{\mathrm{HH}, \text { trans }}=18 \mathrm{~Hz}, 1 \mathrm{H}, \mathrm{ArCH}=\mathrm{CH}_{2}\right), 5.32$ $\left(\mathrm{d},{ }^{3} J_{\mathrm{HH}, c i s}=11 \mathrm{~Hz}, 1 \mathrm{H}, \mathrm{ArCH}=\mathrm{CH}_{2}\right), 4.64$ (pseudo-t, ${ }^{3} J_{\mathrm{HH}}=$ $\left.2 \mathrm{~Hz}, 6 \mathrm{H}, \beta-\mathrm{C}_{5} \mathrm{H}_{4} \mathrm{R}\right), 4.56\left(\mathrm{~s}, 15 \mathrm{H}, \mathrm{C}_{5} H_{5}\right), 4.52$ (pseudo-t, ${ }^{3} \mathrm{~J}_{\mathrm{HH}}=2$ $\mathrm{Hz}, 6 \mathrm{H}, \alpha-\mathrm{C}_{5} \mathrm{H}_{4} \mathrm{R}$ ), 3.94 (d, ${ }^{2} J_{\mathrm{HP}}=15 \mathrm{~Hz}, 2 \mathrm{H}, \mathrm{PCH} \mathrm{H}_{2} \mathrm{Ar}$ ), 2.49-2.25 $\left(\mathrm{m}, \quad 12 \mathrm{H}, \mathrm{C}_{5} \mathrm{H}_{4} \mathrm{CH}_{2} \mathrm{CH}_{2}\right.$ and $\left.\mathrm{C}_{5} \mathrm{H}_{4} \mathrm{CH}_{2} \mathrm{CH}_{2}\right) .{ }^{13} \mathrm{C}\left\{{ }^{1} \mathrm{H}\right\} \quad \mathrm{NMR}$ $\left(\mathrm{DMSO}-d_{6}\right): \delta 137.1(\mathrm{~s}), 135.8(\mathrm{~s}), 130.3(\mathrm{~s}), 128.5\left(\mathrm{~d}, J_{\mathrm{CP}}=8 \mathrm{~Hz}\right)$, $127.0(\mathrm{~s}), 120.6\left(\mathrm{q}, J_{\mathrm{CF}}=321 \mathrm{~Hz}\right), 115.2(\mathrm{~s}), 91.0\left(\mathrm{~d}, J_{\mathrm{CP}}=17 \mathrm{~Hz}\right)$, 70.6 (s), $70.4(\mathrm{~s}), 69.7(\mathrm{~s}), 25.3\left(\mathrm{~d}, J_{\mathrm{CP}}=44 \mathrm{~Hz}\right), 20.3(\mathrm{~s}), 20.0(\mathrm{~d}$, $\left.J_{\mathrm{CP}}=45 \mathrm{~Hz}\right) \cdot{ }^{19} \mathrm{~F}$ NMR (DMSO- $\left.d_{6}\right): \delta-77.7(\mathrm{~s}) \cdot{ }^{31} \mathrm{P}\left\{{ }^{1} \mathrm{H}\right\} \mathrm{NMR}$ $\left(\right.$ DMSO- $\left.d_{6}\right): \delta 30.8(\mathrm{~s})$. FT-IR: $3094(\mathrm{w}), 3013(\mathrm{w}), 2911(\mathrm{w}), 1630$ (w), 1512 (w), 1409 (w), 1261 (s), 1159 (m), 1101 (w), 1030 (s), 997 (w), 917 (w), $810(\mathrm{~m}), 755$ (s), 637 (s) $\mathrm{cm}^{-1}$. UV-vis (THF): $\lambda_{\text {max }} 286 \mathrm{~nm}\left(\varepsilon=1970 \mathrm{M}^{-1} \mathrm{~cm}^{-1}\right), 296 \mathrm{~nm}\left(\varepsilon=1510 \mathrm{M}^{-1} \mathrm{~cm}^{-1}\right)$, $315 \mathrm{~nm}\left(\varepsilon=910 \mathrm{M}^{-1} \mathrm{~cm}^{-1}\right)$. Mass Spec. (ESI, +ve mode): exact mass calculated for $\left[\mathrm{C}_{45} \mathrm{H}_{48}{ }^{96} \mathrm{Ru}^{99} \mathrm{Ru}^{100} \mathrm{RuP}\right]^{+}$: 914.0671; exact 
mass found: 914.0664; difference: $-0.8 \mathrm{ppm}$. Anal. Calcd (\%) for $\mathrm{C}_{46} \mathrm{H}_{48} \mathrm{~F}_{3} \mathrm{O}_{3} \mathrm{PRu}_{3} \mathrm{~S}$ : C, 51.53; H, 4.51. Found: C, 51.84; $\mathrm{H}$, 4.62.

\section{Representative procedure for the preparation of polyelectrolytes 8a-d}

Polyelectrolyte 8a $(3 \times$ Fc). In a grease-free Schlenk flask, monomer $7 \mathrm{a}(0.25 \mathrm{~g}, 0.27 \mathrm{mmol})$ was dissolved in $1.00 \mathrm{~mL}$ of a THF stock solution containing AIBN (0.2 mg, $0.001 \mathrm{mmol})$. The resulting solution was degassed during 3 freeze-pumpthaw cycles before the flask was sealed and the solution was stirred at $85{ }^{\circ} \mathrm{C}$ for $16 \mathrm{~h}$. After cooling to room temperature, the polymerization mixture was poured into diethyl ether and the solids were separated by centrifugation before they were collected, dissolved in a minimum amount of $\mathrm{CH}_{2} \mathrm{Cl}_{2}$, and precipitated in diethyl ether. This precipitation/centrifugation process was repeated once more in diethyl ether and pentane. The polyelectrolyte 8a was dried in vacuo at $50{ }^{\circ} \mathrm{C}$ for $16 \mathrm{~h}$ to yield a yellow powder. Yield $=0.25 \mathrm{~g}, 98 \% .{ }^{1} \mathrm{H}$ NMR (DMSO- $d_{6}$, $\left.125{ }^{\circ} \mathrm{C}\right): \delta 6.94(\mathrm{~s}, \mathrm{br}, 2 \mathrm{H}$, aryl $\mathrm{CH}), 6.19(\mathrm{~s}, \mathrm{br}, 2 \mathrm{H}$, aryl $\mathrm{CH}$ ), 4.17 (s, br, $27 \mathrm{H}, \alpha-\mathrm{C}_{5} H_{4} \mathrm{R}, \beta-\mathrm{C}_{5} H_{4} \mathrm{R}$, and $\left.\mathrm{C}_{5} H_{5}\right), 3.77(\mathrm{~s}, \mathrm{br}, 2 \mathrm{H}$, $\mathrm{PCH}_{2} \mathrm{Ar}$ ), 2.61 (s, br, 6H, $\mathrm{C}_{5} \mathrm{H}_{4} \mathrm{CH}_{2} \mathrm{CH}_{2}$ ), 2.45 (s, br, 6H, $\mathrm{C}_{5} \mathrm{H}_{4} \mathrm{CH}_{2} \mathrm{CH}_{2}$, overlaps with residual $\mathrm{CD}_{3} \mathrm{CD}_{2} \mathrm{HSO}$ signal), 1.96 (s, $1 \mathrm{H}, \mathrm{br}, \mathrm{ArCHCH}$ ), and 1.32 (s, br, 2H, $\mathrm{ArCHCH}_{2}$ ). ${ }^{19} \mathrm{~F}$ NMR (DMSO- $\left.d_{6}, 125{ }^{\circ} \mathrm{C}\right): \delta-77.1(\mathrm{~s}) .{ }^{31} \mathrm{P}\left\{{ }^{1} \mathrm{H}\right\}$ NMR (DMSO- $d_{6}, 125^{\circ} \mathrm{C}$ ): $\delta 31.1$ (s). FT-IR: $3094(\mathrm{w}), 2949(\mathrm{w}), 2919(\mathrm{w}), 1510(\mathrm{w}), 1410(\mathrm{w})$, 1261 (s), 1159 (m), 1105 (w), 1030 (s), 1001 (w), 822 (m), 756 (s), $637(\mathrm{~s}) \mathrm{cm}^{-1}$. UV-vis (THF): $\lambda_{\max } 325 \mathrm{~nm}\left(\varepsilon=260 \mathrm{M}^{-1} \mathrm{~cm}^{-1}\right)$, $436 \mathrm{~nm}\left(\varepsilon=330 \mathrm{M}^{-1} \mathrm{~cm}^{-1}\right)$. GPC (DMF, $0.02 \mathrm{M}\left[n-\mathrm{Bu}_{4} \mathrm{~N}\right][\mathrm{OTf}]$, $60{ }^{\circ} \mathrm{C}$, conventional calibration $v s$. PS standards): $M_{\mathrm{n}}=46900$, $M_{\mathrm{w}}=148000 \mathrm{~g} \mathrm{~mol}^{-1}, Ð=3.16$.

Polyelectrolyte $8 \mathbf{b}(2 \times \mathbf{F c}, 1 \times \mathbf{R c})$. From monomer $7 \mathbf{b}(0.25 \mathrm{~g}$, $0.26 \mathrm{mmol}$ ) and AIBN $(0.2 \mathrm{mg}, 0.001 \mathrm{mmol})$. Yield $=0.24 \mathrm{~g}$, 96\%. ${ }^{1} \mathrm{H}$ NMR (DMSO- $d_{6}, 125{ }^{\circ} \mathrm{C}$ ): $\delta 6.94$ (s, br, 2H, aryl $\mathrm{CH}$ ), $6.21(\mathrm{~s}, \mathrm{br}, 2 \mathrm{H}$, aryl $\mathrm{CH}), 4.63\left[\mathrm{~s}, \mathrm{br}, 2 \mathrm{H}, \beta-\mathrm{C}_{5} H_{4} \mathrm{R}(\mathrm{Ru})\right], 4.56$ $\left[\mathrm{s}, \mathrm{br}, 7 \mathrm{H}, \mathrm{C}_{5} H_{5}(\mathrm{Ru})\right.$ and $\left.\alpha-\mathrm{C}_{5} H_{5} \mathrm{R}(\mathrm{Ru})\right], 4.17[\mathrm{~s}, \mathrm{br}, 18 \mathrm{H}$, $\alpha-\mathrm{C}_{5} H_{4} \mathrm{R}(\mathrm{Fe}), \beta-\mathrm{C}_{5} H_{4} \mathrm{R}(\mathrm{Fe})$, and $\left.\mathrm{C}_{5} H_{5}(\mathrm{Fe})\right], 3.75$ (s, br, $2 \mathrm{H}$, $\mathrm{PCH}_{2} \mathrm{Ar}$ ), 2.61 (s, br, $\left.6 \mathrm{H}, \mathrm{C}_{5} \mathrm{H}_{4} \mathrm{CH}_{2} \mathrm{CH}_{2}\right), 2.42(\mathrm{~s}, \mathrm{br}, 6 \mathrm{H}$, $\mathrm{C}_{5} \mathrm{H}_{4} \mathrm{CH}_{2} \mathrm{CH}_{2}$, overlaps with residual $\mathrm{CD}_{3} \mathrm{CD}_{2} \mathrm{HSO}$ signal), 1.96 (s, br, $1 \mathrm{H}, \mathrm{ArCHCH}$ ), and 1.34 (s, br, 2H, $\mathrm{ArCHCH}_{2}$ ). ${ }^{19} \mathrm{~F} \mathrm{NMR}$ (DMSO- $d_{6}, 125{ }^{\circ} \mathrm{C}$ ): $\delta-76.3$ (s). ${ }^{31} \mathrm{P}\left\{{ }^{1} \mathrm{H}\right\}$ NMR (DMSO- $d_{6}$, $\left.125{ }^{\circ} \mathrm{C}\right): \delta 30.9(\mathrm{~s})$. FT-IR: $3093(\mathrm{w}), 3013(\mathrm{w}), 2916(\mathrm{w}), 1510$ (w), $1410(\mathrm{w}), 1260(\mathrm{~m}), 1221(\mathrm{~m}), 1105(\mathrm{w}), 1030(\mathrm{~s}), 1000(\mathrm{w})$, $814(\mathrm{w}), 772(\mathrm{~s}), 637(\mathrm{~m}) \mathrm{cm}^{-1}$. UV-vis (THF): $\lambda_{\max } 320 \mathrm{~nm}(\varepsilon=$ $\left.440 \mathrm{M}^{-1} \mathrm{~cm}^{-1}\right), 431 \mathrm{~nm}\left(\varepsilon=230 \mathrm{M}^{-1} \mathrm{~cm}^{-1}\right)$. GPC (DMF, $0.02 \mathrm{M}$ $\left[n-\mathrm{Bu}_{4} \mathrm{~N}\right][\mathrm{OTf}], 60{ }^{\circ} \mathrm{C}$, conventional calibration vs. PS standards): $M_{\mathrm{n}}=45100, M_{\mathrm{w}}=184900 \mathrm{~g} \mathrm{~mol}^{-1}, D=4.10$.

Polyelectrolyte 8 c $(1 \times$ Fc, $2 \times$ Rc). From monomer 7c $(0.25 \mathrm{~g}$, $0.24 \mathrm{mmol}$ ) and AIBN (0.2 $\mathrm{mg}, 0.001 \mathrm{mmol})$. Yield $=0.24 \mathrm{~g}$, 97\%. ${ }^{1} \mathrm{H}$ NMR (DMSO- $d_{6}, 125{ }^{\circ} \mathrm{C}$ ): $\delta 6.97$ (s, br, 2H, aryl $\mathrm{CH}$ ), 6.21 (s, br, 2H, aryl $\mathrm{CH}$ ), $4.63\left[\mathrm{~s}, \mathrm{br}, 4 \mathrm{H}, \beta-\mathrm{C}_{5} H_{4} \mathrm{R}(\mathrm{Ru})\right], 4.56$ $\left[\mathrm{s}, \mathrm{br}, 14 \mathrm{H}, \mathrm{C}_{5} H_{5}(\mathrm{Ru})\right.$ and $\left.\alpha-\mathrm{C}_{5} H_{5} \mathrm{R}(\mathrm{Ru})\right], 4.17$ [s, br, 9H, $\alpha-\mathrm{C}_{5} \mathrm{H}_{4} \mathrm{R}(\mathrm{Fe}), \quad \beta-\mathrm{C}_{5} \mathrm{H}_{4} \mathrm{R}(\mathrm{Fe})$, and $\left.\mathrm{C}_{5} \mathrm{H}_{5}(\mathrm{Fe})\right], 3.78$ (s, br, $2 \mathrm{H}$, $\mathrm{PCH}_{2} \mathrm{Ar}$ ), $2.62\left(\mathrm{~s}, \mathrm{br}, 6 \mathrm{H}, \mathrm{C}_{5} \mathrm{H}_{4} \mathrm{CH}_{2} \mathrm{CH}_{2}\right), 2.42(\mathrm{~s}, \mathrm{br}, 6 \mathrm{H}$, $\mathrm{C}_{5} \mathrm{H}_{4} \mathrm{CH}_{2} \mathrm{CH}_{2}$, overlaps with residual $\mathrm{CD}_{3} \mathrm{CD}_{2} \mathrm{HSO}$ signal), 1.90 (s, br, $1 \mathrm{H}, \mathrm{ArCHCH}_{2}$ ), and 1.34 (s, br, 2H, $\operatorname{ArCHCH}_{2}$ ). ${ }^{19} \mathrm{~F} \mathrm{NMR}$ $\left(\right.$ DMSO- $\left.d_{6}, 125{ }^{\circ} \mathrm{C}\right): \delta-76.3(\mathrm{~s}) .{ }^{31} \mathrm{P}\left\{{ }^{1} \mathrm{H}\right\}$ NMR (DMSO- $d_{6}$, $\left.125{ }^{\circ} \mathrm{C}\right): \delta 30.8(\mathrm{~s})$. FT-IR: $3095(\mathrm{w}), 3014(\mathrm{w}), 2916(\mathrm{w}), 1510$ (w), $1410(\mathrm{w}), 1260(\mathrm{~m}), 1224(\mathrm{~m}), 1101$ (w), $1030(\mathrm{~s}), 999(\mathrm{w})$, $811(\mathrm{~m}), 756(\mathrm{~s}), 637(\mathrm{~s}) \mathrm{cm}^{-1}$. UV-vis (THF): $\lambda_{\max } 320 \mathrm{~nm}(\varepsilon=$ $\left.620 \mathrm{M}^{-1} \mathrm{~cm}^{-1}\right), 434 \mathrm{~nm}\left(\varepsilon=150 \mathrm{M}^{-1} \mathrm{~cm}^{-1}\right)$. GPC (DMF, $0.02 \mathrm{M}$ $\left[n-\mathrm{Bu}_{4} \mathrm{~N}\right][\mathrm{OTf}], 60{ }^{\circ} \mathrm{C}$, conventional calibration vs. PS standards): $M_{\mathrm{n}}=69100, M_{\mathrm{w}}=278100 \mathrm{~g} \mathrm{~mol}^{-1}, \emptyset=4.02$.

Polyelectrolyte 8d $(3 \times \mathbf{R c})$. From monomer 7d $(0.25 \mathrm{~g}$, $0.23 \mathrm{mmol}$ ) and AIBN (0.2 $\mathrm{mg}, 0.001 \mathrm{mmol})$. Yield $=0.24 \mathrm{~g}$, 97\%. ${ }^{1} \mathrm{H}$ NMR (DMSO- $d_{6}, 125{ }^{\circ} \mathrm{C}$ ): $\delta 6.98$ (s, br, $2 \mathrm{H}$, aryl $\mathrm{CH}$ ), 6.26 (s, br, 2H, aryl $\mathrm{CH}$ ), 4.63 (s, br, 6H, $\left.\beta-\mathrm{C}_{5} H_{4} \mathrm{R}\right), 4.56(\mathrm{~s}, \mathrm{br}$, $21 \mathrm{H}, \mathrm{C}_{5} H_{5}$, and $\alpha-\mathrm{C}_{5} H_{5} \mathrm{R}$ ), 3.80 (s, br, $2 \mathrm{H}, \mathrm{PCH} \mathrm{H}_{2} \mathrm{Ar}$ ), 2.43 (s, br, $12 \mathrm{H}, \mathrm{C}_{5} \mathrm{H}_{4} \mathrm{CH}_{2} \mathrm{CH}_{2}$ and $\left.\mathrm{C}_{5} \mathrm{H}_{4} \mathrm{CH}_{2} \mathrm{CH}_{2}\right), 1.85(\mathrm{~s}, \mathrm{br}, 1 \mathrm{H}$, $\mathrm{ArCHCH}_{2}$ ), and 1.37 (s, br, 2H, $\left.\mathrm{ArCHCH}_{2}\right) .{ }^{19} \mathrm{~F}$ NMR (DMSO- $d_{6}$, $\left.125{ }^{\circ} \mathrm{C}\right): \quad \delta-76.5$ (s). ${ }^{31} \mathrm{P}\left\{{ }^{1} \mathrm{H}\right\} \quad \mathrm{NMR}$ (DMSO- $\left.d_{6}, 125{ }^{\circ} \mathrm{C}\right)$ : $\delta 30.8$ (s). FT-IR: 3095 (w), 3013 (w), $2914(\mathrm{w}), 1510(\mathrm{w}), 1409$ (w), 1261 (s), 1224 (m), 1160 (m), 1101 (m), 1030 (s), 997 (w), $810(\mathrm{~m}), 755(\mathrm{~m})$, and $637(\mathrm{~s}) \mathrm{cm}^{-1}$. UV-vis (THF): $\lambda_{\max } 315 \mathrm{~nm}$ $\left(\varepsilon=870 \mathrm{M}^{-1} \mathrm{~cm}^{-1}\right)$. GPC (DMF, $0.02 \mathrm{M}\left[n-\mathrm{Bu}_{4} \mathrm{~N}\right][\mathrm{OTf}], 60^{\circ} \mathrm{C}$, conventional calibration vs. PS standards): $M_{\mathrm{n}}=38650, M_{\mathrm{w}}=$ $143450 \mathrm{~g} \mathrm{~mol}{ }^{-1}, D=3.71$.

\section{Results}

\section{Synthesis and characterization}

Reaction of phosphines $5 \mathbf{a}-\mathbf{d}^{13}$ with a slight excess of 4-vinylbenzyl chloride and heating at $75{ }^{\circ} \mathrm{C}$ afforded phosphonium chloride monomers 6a-d in quantitative yields (Scheme 1). Due to the poor solubility of phosphines $\mathbf{5 a - d}$ in DMF, they were initially dissolved in a minimum amount of THF and later combined with DMF, an effective solvent for the quaternization reaction. Butylated hydroxytoluene (BHT) was added to prevent the undesired polymerization of the styrene groups during the quaternization reaction. To improve the solubility of the phosphonium salts in organic solvents and to prevent metallocene degradation, ${ }^{2 k}$ the chloride counter anions were exchanged with triflate anions to quantitatively afford monomers $\mathbf{7 a - d}$. The structure and purity of the monomers were confirmed using multinuclear NMR spectroscopy, IR and UV-vis absorption spectroscopy, mass spectrometry, elemental analysis, and X-ray crystallography (Fig. 1, S1-S16† and Table 2).

Phosphonium triflate monomers $7 \mathbf{a}-\mathbf{d}$ gave rise to a singlet in their ${ }^{19} \mathrm{~F}$ NMR spectra at $\delta \sim-77$ and a singlet in their ${ }^{31} \mathrm{P}\left\{{ }^{1} \mathrm{H}\right\}$ NMR spectra at $\delta \sim 31.0$. Single crystals of monomer 7a suitable for X-ray crystallography were grown by slow evaporation of the solvent from a THF solution (Fig. 1 and Table 1). In the solid-state structure, each tetrahedral phosphonium cation was in close proximity to a triflate anion (shortest contact: $\mathrm{P} 1-\mathrm{O} 23.958 \AA$ ). The $\mathrm{C}-\mathrm{P}$ bond lengths ranged from $1.782(6)$ to $1.803(5) \AA$, and are shorter than those of the parent phosphine $5 \mathbf{a}[1.843(1)$ to $1.855(1) \AA] .{ }^{2 k}$ The average C-P-C angle was $109.5(3)^{\circ}$ and the $\mathrm{C} 44-\mathrm{C} 45$ bond length for the vinyl group was $1.162(9) \AA$.

Using azobisisobutyronitrile (AIBN) as an initiator, the phosphonium triflate monomers $7 \mathbf{a}-\mathbf{d}$ were polymerized in 


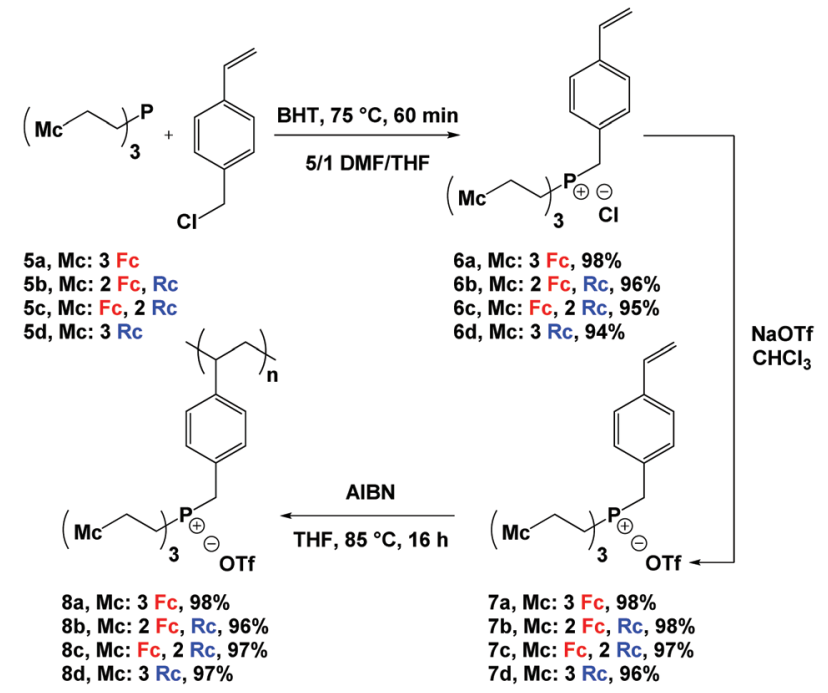

Scheme 1 Synthetic pathway for the preparation of polyelectrolytes $8 a-d$.

THF before they were precipitated into diethyl ether to yield the corresponding polyelectrolytes 8a-d (Scheme 1). At room temperature, these polyelectrolytes gave rise to very broad ${ }^{1} \mathrm{H}$, ${ }^{31} \mathrm{P}\left\{{ }^{1} \mathrm{H}\right\}$, and ${ }^{19} \mathrm{~F}$ NMR spectra. However, upon heating to $125{ }^{\circ} \mathrm{C}$ the spectra sharpened (Fig. S17-S20†). Disappearance of the vinyl proton resonances and the observation of broad peaks associated with the unsaturated polyelectrolyte backbone $(\delta 0.75-2.15)$ in the ${ }^{1} \mathrm{H}$ NMR spectra of the polyelectrolytes confirmed successful polymerization. The presence of the aromatic $(\delta$ 5.75-7.50), metallocene $(\delta$ 4.00-5.00), methylene bridge ( $\delta 3.40-4.20)$, and ethylene bridge $(\delta 2.10-3.25)$ proton resonances in the ${ }^{1} \mathrm{H}$ NMR spectra; a peak for each polyelectrolyte $(\delta \sim 31.0)$ in the ${ }^{31} \mathrm{P}\left\{{ }^{1} \mathrm{H}\right\}$ NMR spectra; and a peak for each polyelectrolyte $(\delta \sim-77)$ in the ${ }^{19} \mathrm{~F}$ NMR spectra further supported the proposed structures of the polyelectrolytes.

Gel permeation chromatography (GPC) experiments were carried out to evaluate the molecular weight distributions for polyelectrolytes $\mathbf{8 a - d}$. To overcome common issues regarding strong interactions between polyelectrolytes and GPC columns, a $60{ }^{\circ} \mathrm{C}$ DMF solution containing $0.02 \mathrm{M}\left[n-\mathrm{Bu}_{4} \mathrm{~N}\right][\mathrm{OTf}]$ was

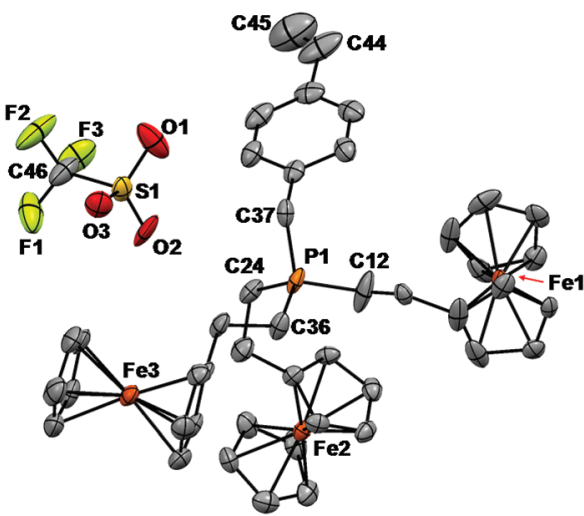

Fig. 1 Solid-state structure of monomer 7a. Anisotropic displacement ellipsoids are shown at $50 \%$ probability and hydrogen atoms have been omitted for clarity. Selected bond lengths (Å): P1-C12 1.782(6), P1-C24 1.803(5), P1-C36 1.798(5), P1-C37 1.791(6), C44-C45 1.162(9). Selected bond angles (degrees): C12-P1-C24 111.7(3), C12-P1-C36 108.5(3), C12-P1-C37 110.9(3), C24-P1-C36 110.0(3), C24-P1-C37 106.2(2), and C36-P1-C37 109.6(3).

used as an eluent in tandem with Teflon-treated size-exclusion columns. The results confirmed the macromolecular nature of the polyelectrolytes 8a-d $\left(M_{\mathrm{n}}: 38650-69100 \mathrm{gmol}^{-1}, \quad D\right.$ : 3.16-4.10, Tables 2 and S1, and Fig. S21†).

\section{UV-vis absorption spectroscopy and cyclic voltammetry}

Similar to phosphines $\mathbf{5 a - d},{ }^{13}$ the ferrocene units in polyelectrolytes 8a-d gave rise to two absorption maxima at $c a .434 \mathrm{~nm}$ and $320 \mathrm{~nm}$ while the ruthenocene units exhibited a single absorption maximum at $c a .320 \mathrm{~nm}$. The relative intensities of each peak varied with the number of ferrocene/ruthenocene units present (Table 2 and Fig. S22-S24†).

The electrochemical properties of the phosphonium triflate monomers $7 \mathbf{a}-\mathbf{d}$ and the corresponding polyelectrolytes 8a-d were examined by cyclic voltammetry $(\mathrm{CV})$ in a $\mathrm{CH}_{2} \mathrm{Cl}_{2} / \mathrm{CH}_{3} \mathrm{CN}$ (2/1) solvent mixture containing $0.1 \mathrm{M}\left[n-\mathrm{Bu}_{4} \mathrm{~N}\right][\mathrm{OTf}]$ as supporting electrolyte. Consistent with the electrochemical properties of the parent phosphines, ${ }^{13}$ the ferrocene units of the monomers and polyelectrolytes were oxidized reversibly,

Table 2 Selected characterization data for monomers $7 a-d$ and polyelectrolytes $8 a-d$

\begin{tabular}{|c|c|c|c|c|c|c|c|c|}
\hline Compound & ${ }^{31} \mathrm{P}$ NMR shift ${ }^{a}(\delta)$ & $\varepsilon, 320 \mathrm{~nm}^{b}\left(\mathrm{M}^{-1} \mathrm{~cm}^{-1}\right)$ & $\varepsilon, 434 \mathrm{~nm}^{b}\left(\mathrm{M} \mathrm{cm}^{-1}\right)$ & $E_{\mathrm{pa}, \mathrm{Rc}}{ }^{c, d}(\mathrm{mV})$ & $E_{1 / 2, \mathrm{Fc}}{ }^{c}(\mathrm{mV})$ & $M_{\mathrm{n}}^{e}\left(\mathrm{~g} \mathrm{~mol}^{-1}\right)$ & $\mathrm{DP}_{\mathrm{n}}{ }^{e}$ & $\bigoplus^{e}$ \\
\hline $7 \mathbf{b}$ & 31.0 & 440 & 240 & 465 & 15 & - & - & - \\
\hline $8 a$ & 31.1 & 260 & 330 & - & 20 & 46900 & 50 & 3.16 \\
\hline $8 b$ & 30.9 & 440 & 230 & - & 20 & 45100 & 46 & 4.10 \\
\hline $8 c$ & 30.8 & 620 & 150 & 410 & 35 & 69100 & 67 & 4.02 \\
\hline
\end{tabular}

${ }^{a}$ Recorded in DMSO- $d_{6}$ and referenced relative to triphenylphosphine internal standard. ${ }^{b}$ Recorded in THF. ${ }^{c}$ Recorded at a scan rate of 250 $\mathrm{mV} \mathrm{s}^{-1}$ in degassed $\mathrm{CH}_{2} \mathrm{Cl}_{2} / \mathrm{CH}_{3} \mathrm{CN}(2 / 1)$ solutions of the analyte containing $0.1 \mathrm{M}\left[n-\mathrm{Bu}_{4} \mathrm{~N}\right][\mathrm{OTf}]$ as supporting electrolyte and referenced relative to the ferrocene/ferrocenium redox couple. ${ }^{d}$ Irreversible process; anodic peak potential reported. ${ }^{e}$ Determined by conventional calibration GPC vs. polystyrene standards in DMF $\left(60^{\circ} \mathrm{C}\right)$ containing $0.02 \mathrm{M}\left[n-\mathrm{Bu}_{4} \mathrm{~N}\right][\mathrm{OTf}]$. 
while the ruthenocene moieties gave rise to an irreversible oxidation wave. The observed irreversible behavior was consistent with the ability of ruthenocenium cations to rapidly engage in electrochemically-induced reactions. ${ }^{5 d, 18}$ Due to the presence of the cationic phosphonium centers in the monomers, the recorded $E_{1 / 2, \mathrm{Fc}}$ and $E_{\mathrm{pa}, \mathrm{Rc}}$ values were slightly more positive than those of the parent phosphines (Table 2, Fig. S25 and $\mathrm{S} 26 \dagger) \cdot{ }^{13}$

\section{Thermal analysis and pyrolysis studies}

Differential scanning calorimetry (DSC) studies of polyelectrolytes 8a-d revealed glass transition temperatures $\left(T_{\mathrm{g}}\right)$ between 165 and $177^{\circ} \mathrm{C}$ (Fig. S27† and Table 3). TGA studies demonstrated that polyelectrolytes 8a-d have high thermal stability, with the onset of decomposition observed at $c a .310{ }^{\circ} \mathrm{C}$ and char yields ranging from 33 to $54 \%$ (Fig. 2 and Table 3 ).

Based on the high char yields observed and growing interest in the catalytic and electrochemical properties of nanostructured metal phosphides ${ }^{19}$ we decided to explore the preceramic behavior of polyelectrolytes 8a-d. Polyelectrolyte films with approximate thickness of $6 \mu \mathrm{m}$ (Fig. S28 $\dagger$ ) were prepared by drop casting and pyrolyzed at $1000{ }^{\circ} \mathrm{C}$ under a flow of $\mathrm{N}_{2} / \mathrm{H}_{2}$ (95/5) for $3 \mathrm{~h}$. Each pyrolysis experiment was repeated in triplicate, and representative SEM images and relevant data are shown in Fig. 3 and Table 3. It is worth noting that we were unable to employ transmission electron microscopy (TEM) for our studies as our thorough attempts to dislodge the nanomaterials produced by pyrolysis from silicon substrates using physical scraping (razor blade), ultrasonication, and solvent rinsing were unsuccessful. However, our SEM analyses provided significant insight into the structures of the nanomaterials produced. In each case, pyrolysis of polyelectrolyte films resulted in the formation of large particles and/or continuous materials surrounded by numerous smaller particles. In the case of the nanomaterials produced from $\mathbf{8 b}$ and $\mathbf{8 c}$, the multi-faceted appearance of the imaged materials hinted to the fact that they may be crystalline (vide infra).

Elemental maps (EDX spectroscopy) revealed that the nanomaterials produced were comprised of $\mathrm{C}, \mathrm{O}, \mathrm{P}, \mathrm{Fe}$, and/or

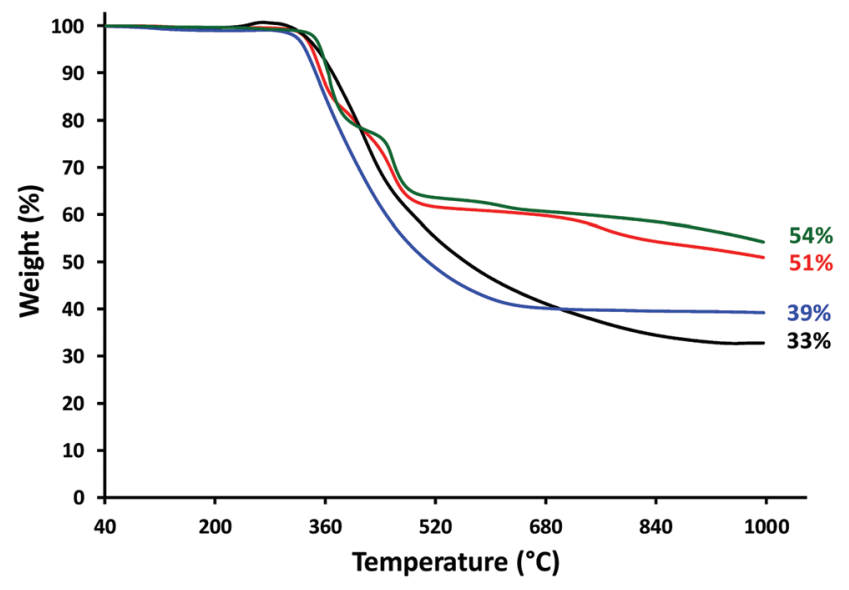

Fig. 2 TGA data obtained for polyelectrolytes: $8 \mathbf{a}(3 \times \mathrm{Fc}$, black), $8 \mathbf{b}$ $(2 \times \mathrm{Fc}, 1 \times \mathrm{Rc}$; red), $8 \mathrm{c}(1 \times \mathrm{Fc}, 2 \times \mathrm{Rc}$; blue), and $8 \mathrm{~d}(3 \times \mathrm{Rc}$, green).
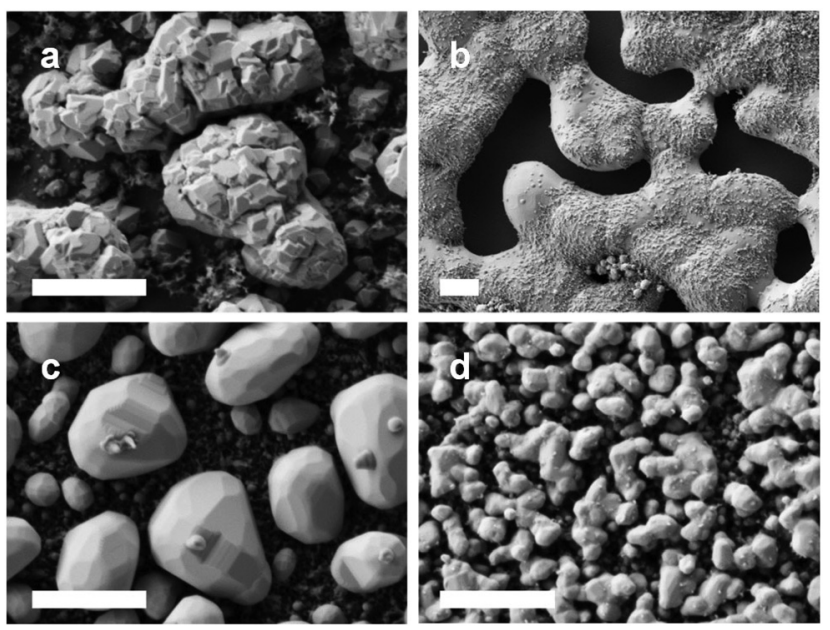

Fig. 3 SEM images of the nanomaterials prepared via the pyrolysis of films of polyelectrolytes (a) $8 a$, (b) $8 b$, (c) $8 c$, and (d) $8 d$. Scale bars $=$ $1 \mu \mathrm{m}$.

Table 3 Thermal characterization and elemental composition data for the nanomaterials produced via the pyrolysis of polyelectrolytes $8 a-d^{a}$

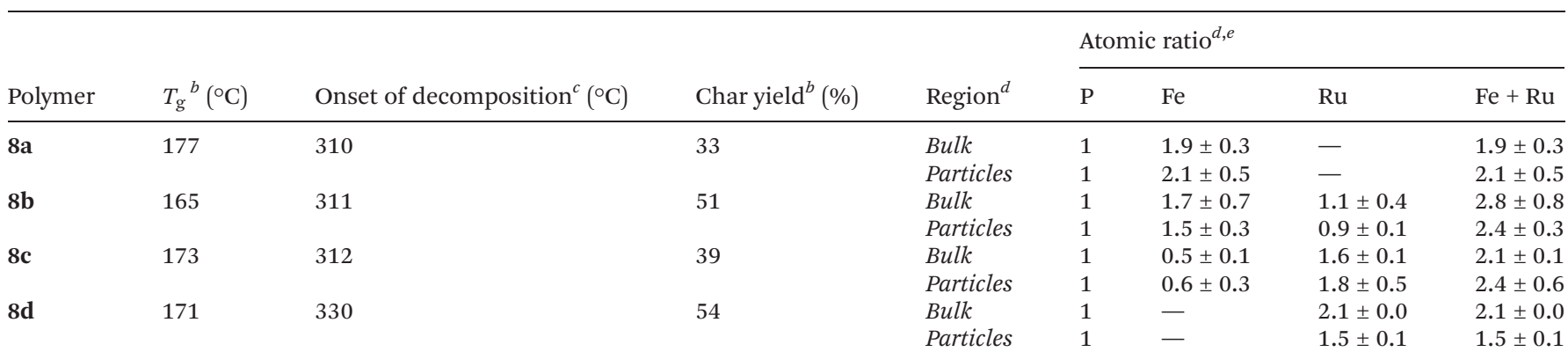

${ }^{a}$ Carried out at $1000{ }^{\circ} \mathrm{C}$ under $\mathrm{N}_{2} / \mathrm{H}_{2}(95 / 5)$ gas mixture as the carrier gas at an approximate flow rate of $c a .60 \mathrm{~mL}$ min ${ }^{-1} .{ }^{b}$ Determined using DSC. ${ }^{c}$ Determined using TGA. ${ }^{d}$ Atomic ratios determined using EDX spectroscopy for dense regions of relatively large particles ('bulk') and less dense regions of relatively small particles ('particles'). See ESI for additional details. ${ }^{e}$ Phosphorus stoichiometry fixed at 1. 

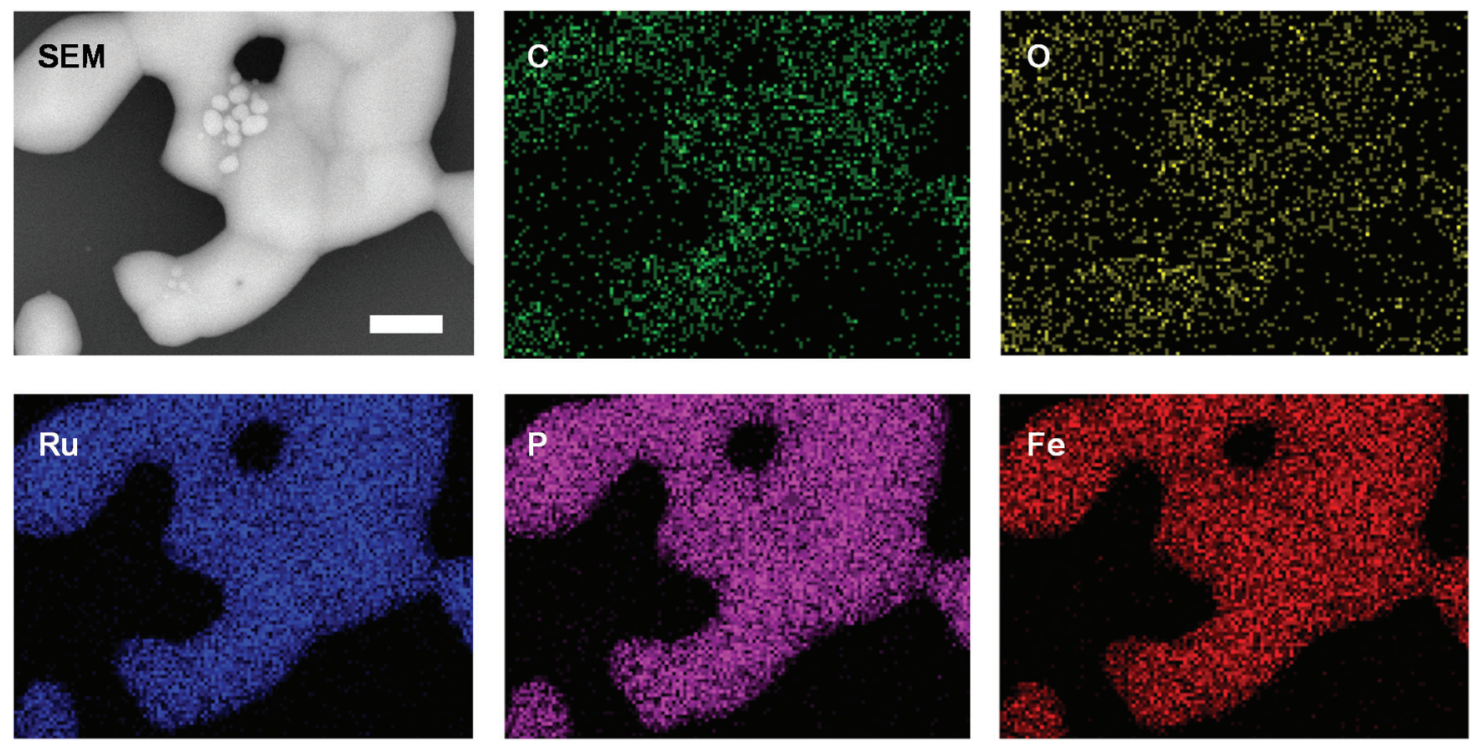

Fig. 4 SEM images and elemental maps $(\mathrm{O}, \mathrm{Ru}, \mathrm{Fe}, \mathrm{P}, \mathrm{C})$ for the nanomaterials prepared via the pyrolysis of a film of polyelectrolyte $8 \mathbf{b}$. Scale bar $=1 \mu \mathrm{m}$.

$\mathrm{Ru}$ and that the inorganic components were distributed throughout the nanomaterials produced (Fig. 4, S29-S31†). The presence of carbon was attributed to incomplete volatilization of the polystyrene backbone and oxidation during brief (and unavoidable) exposure of the samples to air prior to SEM analysis accounts for the presence of oxygen. Unfortunately, the elemental maps obtained provide little quantitative information about the composition of the nanomaterials produced.
With this in mind, at least five different EDX data sets (elemental analyses) were collected for regions $\left(c a .1 \mu \mathrm{m}^{2}\right)$ of the nanomaterial surfaces densely populated with relatively large particles and/or continuous material ('bulk') and with relatively small particles ('particles') for each of the samples (Fig. S32-S35† and Table 3). For each sample, the smaller particles produced were clearly embedded within a carbon-rich matrix, and the overall phosphorus/metal content was signifi-
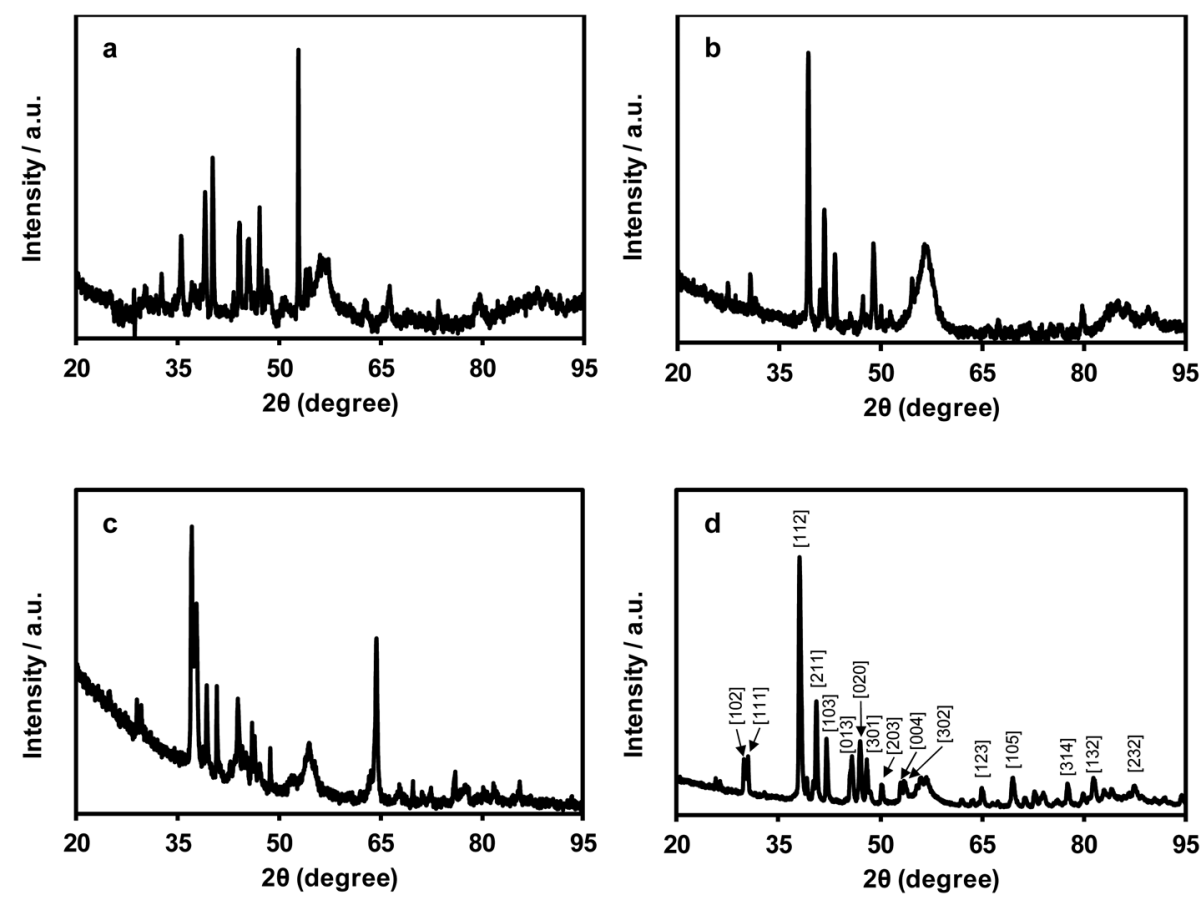

Fig. 5 Powder X-ray diffractograms for the nanomaterials prepared via the pyrolysis of films of polyelectrolytes (a) 8a, (b) 8b, (c) 8c, and (d) 8d. Miller indices corresponding to $\mathrm{Ru}_{2} \mathrm{P}$ are shown in panel (d). 
cantly lower than those observed for areas densely populated with bulk material.

\section{Powder X-ray diffraction studies}

Powder X-ray diffraction studies of the nanomaterials produced by pyrolysis of films of polyelectrolytes $\mathbf{8 a - d}$ are shown in Fig. 5. In each case, the patterns produced confirmed the presence of crystalline materials. Qualitative phase identification studies were performed through careful comparison of our diffraction data with those of a PXRD database, ${ }^{20}$ which included data for known mono- and bimetallic phosphides (i.e., $\mathrm{M}_{4} \mathrm{P}, \mathrm{M}_{3} \mathrm{P}, \mathrm{M}_{2} \mathrm{P}$, and $\mathrm{MP}$; where $\mathrm{M}$ : Fe and/or $\mathrm{Ru}$ ), metal carbides, pure metals, etc. (Fig. S36-S39†). The PXRD data for the films produced from polyelectrolytes $\mathbf{8 a}-\mathbf{c}$ were not closely matched with those of any known phases, and were indicative of the presence of multiple crystalline phases, which prevented us from indexing the data. Conversely, the PXRD data collected for the film produced from polyelectrolyte 8d was consistent with that of $\mathrm{Ru}_{2} \mathrm{P}$ (Fig. $5 \mathrm{~d}$ and $\mathrm{S} 39 \dagger$ ).

\section{Discussion}

Considering the PXRD, EDX spectroscopy, and elemental mapping results obtained for the nanomaterials produced from pyrolyzed films of polyelectrolytes $\mathbf{8 a - c}$, two points become immediately obvious. First, given the large standard deviations associated with the atomic ratios determined from the EDX data collected, it is clear that phosphorus, iron, and/ or ruthenium are not distributed uniformly throughout the nanomaterials produced. It is therefore probable that multiple different materials/phases have been produced. For the heterobimetallic materials derived from $\mathbf{8 b}$ and $\mathbf{8 c}$, PXRD data confirmed the absence of simple $\mathrm{Fe}_{2} \mathrm{P} / \mathrm{Ru}_{2} \mathrm{P} / \mathrm{Fe}_{3} \mathrm{P} / \mathrm{Ru}_{3} \mathrm{P}$ phases and supported this hypothesis. Second, within a single standard deviation, the average composition of the small particles and bulk materials analyzed were the same. Conversely, for the nanomaterials derived from $\mathbf{8 d}$, the relatively smaller standard deviations calculated point toward the uniform distribution of phosphorus and ruthenium throughout regions containing bulk material and relatively small particles. Furthermore, this was the only case where the composition of the bulk $(\mathrm{P}: \mathrm{Ru}$, $1: 2.1 \pm 0.0)$ and small-particle-rich ( $\mathrm{P}: \mathrm{Ru}, 1: 1.5 \pm 0.1)$ were statistically different. When these results are combined with the PXRD data collected and indexed for this sample, it becomes clear that the bulk phase produced from the pyrolysis of films of polyelectrolyte $\mathbf{8 d}$ is comprised of $\mathrm{Ru}_{2} \mathrm{P}$.

\section{Conclusion}

In conclusion, starting from stable tertiary phosphines containing all possible combinations of ethylferrocene and ethylruthenocene substituents, styrene-based phosphonium triflate monomers with four different stoichiometric ratios of $\mathrm{Fe} / \mathrm{Ru}$ were synthesized and fully characterized. Via free-radical polymerization of the triflate salts, four polyelectrolytes were prepared and analyzed. Due to the presence of ferrocene/ruthenocene, these materials exhibited redox properties and gave rise to UV-vis absorption maxima consistent with the number of each metallocene present. GPC and DSC results confirmed the macromolecular nature of the polyelectrolytes and TGA studies confirmed their stability up to $\sim 310^{\circ} \mathrm{C}$. Studies of the nanomaterials that resulted from the pyrolysis of polyelectrolytes 8a-d using SEM and PXRD showed that they can be used as precursors to crystalline nanomaterials. EDX spectroscopy and elemental mapping data indicated that the crystalline nanomaterials contained $\mathrm{Fe}, \mathrm{Ru}$, and $\mathrm{P}$ distributed throughout, with $\mathrm{Fe} / \mathrm{Ru} / \mathrm{P}$ ratios influenced by the polyelectrolyte structures.

\section{Acknowledgements}

The authors acknowledge the University of Western Ontario, the Natural Science and Engineering Research Council (NSERC) of Canada (DG, 435675), the Ontario Ministry of Research and Innovation (ERA, ER14-10-147) and the Canada Foundation for Innovation (JELF, 33977) for funding this work. We thank Prof. Paul Ragogna and Mr Tyler Cuthbert for assistance with phosphine experiments, use of equipment, and helpful discussions. We thank Mr Tim Goldhawk and Dr Todd Simpson of the Western Nanofabrication Facility for assistance with SEM and EDX spectroscopy studies, and Dr Paul Boyle, Ms Stephanie Barbon, Dr Anastasia Colomba, and Mr Joe Paquette for assistance with X-ray diffraction experiments and helpful discussions. We also appreciate the time and effort that Mr Eric Landry and Mr Ahmad Abu Romeh (PolyAnalytik Canada) spent collecting the GPC data presented.

\section{Notes and references}

1 Reviews: (a) C.-L. Ho and W.-Y. Wong, Coord. Chem. Rev., 2011, 255, 2469-2502; (b) G. R. Whittell, M. D. Hager, U. S. Schubert and I. Manners, Nat. Mater., 2011, 10, 176188; (c) D. Astruc, Nat. Chem., 2012, 4, 255-267; (d) C. Friebe, M. D. Hager, A. Winter and U. S. Schubert, Adv. Mater., 2012, 24, 332-345; (e) C. G. Hardy, L. Ren, J. Zhang and C. Tang, Isr. J. Chem., 2012, 52, 230-245; (f) H. Bhattacharjee and J. Müller, Coord. Chem. Rev., 2016, 314, 114-133; $(g)$ Y. Yan, J. Zhang, L. Ren and C. Tang, Chem. Soc. Rev., 2016, 45, 5232-5263.

2 For example: (a) T. J. Peckham, A. J. Lough and I. Manners, Organometallics, 1999, 18, 1030-1040; (b) B. F. G. Johnson, K. M. Sanderson, D. S. Shephard, D. Ozkaya, W. Zhou, H. Ahmed, M. D. R. Thomas, L. Gladden and M. Mantle, Chem. Commun., 2000, 1317-1318; (c) Y. Ma, W.-F. Dong, M. A. Hempenius, H. Mohwald and G. Julius Vancso, Nat. Mater., 2006, 5, 724-729; (d) X. Wang, G. Guerin, H. Wang, Y. Wang, I. Manners and M. A. Winnik, Science, 2007, 317, 644-647; (e) C. Ornelas, J. Ruiz, C. Belin and D. Astruc, 
J. Am. Chem. Soc., 2009, 131, 590-601; $(f)$ H. Zhan, S. Lamare, A. Ng, T. Kenny, H. Guernon, W.-K. Chan, A. B. Djurišić, P. D. Harvey and W.-Y. Wong, Macromolecules, 2011, 44, 5155-5167; (g) Z. M. Al-Badri, R. R. Maddikeri, Y. Zha, H. D. Thaker, P. Dobriyal, R. Shunmugam, T. P. Russell and G. N. Tew, Nat. Commun., 2011, 2, 482; (h) L. Ren, J. Zhang, C. G. Hardy, S. Ma and C. Tang, Macromol. Rapid Commun., 2012, 33, 510-516; (i) R. H. Staff, M. Gallei, M. Mazurowski, M. Rehahn, R. Berger, K. Landfester and D. Crespy, ACS Nano, 2012, 6, 9042-9049; ( $j$ ) X. Wang, K. Cao, Y. Liu, B. Tsang and S. Liew, J. Am. Chem. Soc., 2013, 135, 3399-3402; (k) A. Rabiee Kenaree, B. M. Berven, P. J. Ragogna and J. B. Gilroy, Chem. Commun., 2014, 50, 10714-10717; (l) R. Guterman, A. Rabiee Kenaree, J. B. Gilroy, E. R. Gillies and P. J. Ragogna, Chem. Mater., 2015, 27, 1412-1419; ( $m$ ) J. Zhang, J. Yan, P. Pageni, Y. Yan, A. Wirth, Y.-P. Chen, Y. Qiao, Q. Wang, A. W. Decho and C. Tang, Sci. Rep., 2015, 5, 11914; (n) M. Hadadpour, J. Gwyther, I. Manners and P. J. Ragogna, Chem. Mater., 2015, 27, 3430-3440.

3 (a) S. B. Clendenning, S. Han, N. Coombs, C. Paquet, M. S. Rayat, D. Grozea, P. M. Brodersen, R. N. S. Sodhi, C. M. Yip, Z. H. Lu and I. Manners, Adv. Mater., 2004, 16, 291-296; (b) W. Y. Chan, S. B. Clendenning, A. Berenbaum, A. J. Lough, S. Aouba, H. E. Ruda and I. Manners, J. Am. Chem. Soc., 2005, 127, 1765-1772; (c) K. Liu, S. B. Clendenning, L. Friebe, W. Y. Chan, X. Zhu, M. R. Freeman, G. C. Yang, C. M. Yip, D. Grozea, Z.-H. Lu and I. Manners, Chem. Mater., 2006, 18, 2591-2601; (d) M. Zamora, S. Bruña, B. Alonso and I. Cuadrado, Macromolecules, 2011, 44, 7994-8007.

4 (a) J. B. Gilroy, S. K. Patra, J. M. Mitchels, M. A. Winnik and I. Manners, Angew. Chem., Int. Ed., 2011, 50, 58515855; (b) Y. Zha, H. D. Thaker, R. R. Maddikeri, S. P. Gido, M. T. Tuominen and G. N. Tew, J. Am. Chem. Soc., 2012, 134, 14534-14541; (c) J. Zhang, L. Ren, C. G. Hardy and C. Tang, Macromolecules, 2012, 45, 6857-6863; (d) J. Zhang, Y. Yan, M. W. Chance, J. Chen, J. Hayat, S. Ma and C. Tang, Angew. Chem., Int. Ed., 2013, 52, 13387-13391; (e) J. Zhang, Y. Yan, J. Chen, W. M. Chance, J. Hayat, Z. Gai and C. Tang, Chem. Mater., 2014, 26, 31853190; (f) R. Ciganda, H. Gu, P. Castel, P. Zhao, J. Ruiz, R. Hernández and D. Astruc, Macromol. Rapid Commun., 2016, 37, 105-111.

5 (a) K. Liu, C.-L. Ho, S. Aouba, Y.-Q. Zhao, Z.-H. Lu, S. Petrov, N. Coombs, P. Dube, H. E. Ruda, W.-Y. Wong and I. Manners, Angew. Chem., Int. Ed., 2008, 47, 12551259; (b) B. Bagh, J. B. Gilroy, A. Staubitz and J. Müller, J. Am. Chem. Soc., 2010, 132, 1794-1795; (c) Q. Dong, G. Li, C.-L. Ho, M. Faisal, C.-W. Leung, P. W.-T. Pong, K. Liu, B.-Z. Tang, I. Manners and W.-Y. Wong, Adv. Mater., 2012, 24, 1034-1040; (d) M. Erhard, K. Lam, M. Haddow, G. R. Whittell, W. E. Geiger and I. Manners, Polym. Chem., 2014, 5, 1264-1274; (e) A. D. Russell, G. R. Whittell, M. F. Haddow and I. Manners,
Organometallics, 2014, 33, 5349-5357; $(f)$ Q. Dong, G. Li, H. Wang, P. Wing-Tat Pong, C.-W. Leung, I. Manners, C.-L. Ho, H. Li and W.-Y. Wong, J. Mater. Chem., 2015, 3, 734-741; (g) H. Braunschweig, A. Damme, S. Demeshko, K. Dück, T. Kramer, I. Krummenacher, F. Meyer, K. Radacki, S. Stellwag-Konertz and G. R. Whittell, J. Am. Chem. Soc., 2015, 137, 1492-1500; (h) Z. Meng, K. Sato, T. Sukegawa, K. Oyaizu, C.-L. Ho, J. Xiang, Y.-H. Feng, Y. H. Lo, H. Nishide and W.-Y. Wong, J. Organomet. Chem., 2016, 812, 51-55.

6 (a) J. A. Rodriguez and D. W. Goodman, Science, 1992, 257, 897-903; (b) S. Sun, C. B. Murray, D. Weller, L. Folks and A. Moser, Science, 2000, 287, 1989-1992; (c) D. Astruc, F. Lu and J. R. Aranzaes, Angew. Chem., Int. Ed., 2005, 44, 7852-7872; (d) A.-H. Lu, E. L. Salabas and F. Schüth, Angew. Chem., Int. Ed., 2007, 46, 1222-1244; (e) K. J. Major, C. De and S. O. Obare, Plasmonics, 2009, 4, 61-78; (f) S. Shylesh, V. Schünemann and W. R. Thiel, Angew. Chem., Int. Ed., 2010, 49, 3428-3459; (g) H. Goesmann and C. Feldmann, Angew. Chem., Int. Ed., 2010, 49, 1362-1395; (h) U. Banin, Y. Ben-Shahar and K. Vinokurov, Chem. Mater., 2014, 26, 97-110; (i) P. Buchwalter, J. Rosé and P. Braunstein, Chem. Rev., 2015, 115, 28-126.

7 (a) B. Li, J. Wang, Y. Yuan, H. Ariga, S. Takakusagi and K. Asakura, ACS Catal., 2011, 1, 1521-1528; (b) V. Kelsen, A. Meffre, P.-F. Fazzini, P. Lecante and B. Chaudret, ChemCatChem, 2014, 6, 1714-1720; (c) M. Kaushik, H. M. Friedman, M. Bateman and A. Moores, RSC Adv., 2015, 5, 53207-53210; (d) R. Hudson, V. Chazelle, M. Bateman, R. Roy, C.-J. Li and A. Moores, ACS Sustainable Chem. Eng., 2015, 3, 814-820.

8 J.-Q. Du, Y. Zhang, T. Tian, S.-C. Yan and H.-T. Wang, Mater. Res. Bull., 2009, 44, 1347-1351.

9 (a) G. L. Ott, T. Fleisch and W. N. Delgass, J. Catal., 1979, 60, 394-403; (b) M. C. Bahome, L. L. Jewell, K. Padayachy, D. Hildebrandt, D. Glasser, A. K. Datye and N. J. Coville, Appl. Catal., A, 2007, 328, 243-251.

10 (a) J. P. Wilcoxon and B. L. Abrams, Chem. Soc. Rev., 2006, 35, 1162-1194; (b) N. Semagina and L. Kiwi-Minsker, Catal. Rev.: Sci. Eng., 2009, 51, 147-217.

11 M. Zaheer, T. Schmalz, G. Motz and R. Kempe, Chem. Soc. Rev., 2012, 41, 5102-5116.

12 T. Nishikubo, T. Iizawa, K. Kobayashi and M. Okawara, Tetrahedron Lett., 1981, 22, 3873-3874.

13 A. Rabiee Kenaree, T. J. Cuthbert, S. M. Barbon, P. D. Boyle, E. R. Gillies, P. J. Ragogna and J. B. Gilroy, Organometallics, 2015, 34, 4272-4280.

14 S. v. Bruker-AXS, 2013, Bruker-AXS, Madison, WI 53711, USA.

15 T. v. Bruker-AXS, 2012, Bruker-AXS, Madison, WI 53711, USA.

16 G. M. Sheldrick, Acta Crystallogr., Sect. A: Fundam. Crystallogr., 2015, 71, 3-8.

17 G. M. Sheldrick, Acta Crystallogr., Sect. C: Cryst. Struct. Commun., 2015, 71, 3-8. 
18 (a) S. Trupia, A. Nafady and W. E. Geiger, Inorg. Chem., 2003, 42, 5480-5482; (b) J. C. Swarts, A. Nafady, J. H. Roudebush, S. Trupia and W. E. Geiger, Inorg. Chem., 2009, 48, 2156-2165.

19 (a) H. Teller, O. Krichevski, M. Gur, A. Gedanken and A. Schechter, ACS Catal., 2015, 5, 4260-4267; (b) Y. Shi and B. Zhang, Chem. Soc. Rev., 2016, 45, 1529-1541; (c) A. Berenguer, T. M. Sankaranarayanan, G. Gómez,
I. Moreno, J. M. Coronado, P. Pizarro and D. P. Serrano, Green Chem., 2016, 18, 1938-1951; (d) Y. Lu, T. Wang, X. Li, G. Zhang, H. Xue and H. Pang, RSC Adv., 2016, 6, 87188-87212; (e) J. F. Callejas, C. G. Read, C. W. Roske, N. S. Lewis and R. E. Schaak, Chem. Mater., 2016, 28, 6017-6044.

20 The PXRD patterns were compared using the ICSD database and PDF4+ software. 